\title{
Energy improvement for energy minimizing functions in the complement of generalized Reifenberg-flat sets
}

\author{
ANTOINE LEMENANT
}

\begin{abstract}
Let $P$ be a hyperplane in $\mathbb{R}^{N}$, and denote by $d_{H}$ the Hausdorff distance. We show that for all positive radius $r<1$ there is an $\varepsilon>0$, such that if $K$ is a Reifenberg-flat set in $B(0,1) \subset \mathbb{R}^{N}$ that contains the origin, with $d_{H}(K, P) \leq \varepsilon$, and if $u$ is an energy minimizing function in $B(0,1) \backslash K$ with restricted values on $\partial B(0,1) \backslash K$, then the normalized energy of $u$ in $B(0, r) \backslash K$ is bounded by the normalized energy of $u$ in $B(0,1) \backslash K$. We also prove the same result in $\mathbb{R}^{3}$ when $K$ is an $\varepsilon$-minimal set, that is a generalization of Reifenberg-flat sets with minimal cones of type $\mathbb{Y}$ and $\mathbb{T}$. Moreover, the result is still true for a further generalization of sets called $\left(\varepsilon, \varepsilon_{0}\right)$-minimal. This article is a preliminary study for a forthcoming paper where a regularity result for the singular set of the MumfordShah functional close to minimal cones in $\mathbb{R}^{3}$ is proved by the same author.
\end{abstract}

Mathematics Subject Classification (2010): 49Q20 (primary); 49Q05 (secondary).

\section{Introduction}

Let $B$ be the unit ball in $\mathbb{R}^{N}$ and let $K$ be a closed set with locally finite $\mathcal{H}^{N-1}$ measure. We say that $u$ locally minimizes the energy in $B \backslash K$ if for every ball $B(x, r)$ compactly contained in $B$ and every function $v \in W^{1,2}(B \backslash K)$ such that $v=u$ in $B \backslash(B(x, r) \cup K)$ we have

$$
\int_{B(x, r) \backslash K}|\nabla u(x)|^{2} d x \leq \int_{B(x, r) \backslash K}|\nabla v(x)|^{2} d x .
$$

In other words, $u$ is harmonic in $B \backslash K$ and satisfies a weak mixed boundary value problem, of type Neumann on $K$ and Dirichlet on $\partial B \backslash K$.

If $u$ is a local energy minimizer in $B \backslash K$, we denote by

$$
\omega_{2}(x, r):=\frac{1}{r^{N-1}} \int_{B(x, r) \backslash K}|\nabla u(x)|^{2} d x
$$

the normalized energy of $u$ in $B(x, r)$. It is well known (see for instance [2, Exercice 7.6]) that if $K=\emptyset$, then for a local energy minimizer in $B$ (that means that $u$ 
is harmonic in $B$ ) we have for all $r>0$,

$$
\omega_{2}(0, r) \leq r^{\gamma} \omega_{2}(0,1)
$$

with $\gamma=1$. This follows as a consequence of the proof of the mean value inequality for subharmonic functions, applied to $|\nabla u|^{2}$. By a reflection argument, this is also true if $K$ is a hyperplane in $\mathbb{R}^{N}$ containing 0 . In this paper, we seek some conditions on $K$ that imply (1.1) for all local energy minimizers in $B \backslash K$ and for some positive exponent $\gamma$. For instance, we want to prove that (1.1) is true when $K$ is sufficiently flat, or sufficiently close to a minimal cone. More precisely, if $r_{0}<1$ is a given radius, we will find some conditions on $K$ for which we know that (1.1) is true for any local energy minimizer in $B \backslash K$, at least for $r=r_{0}$ and for a positive exponent $\gamma$.

Notice that for some given $r$ and $\gamma$, we cannot expect that (1.1) hold for any set $K$ at distance arbitrary small to a hyperplane. Indeed, consider a little tube of size $\varepsilon$ in the unit disc of $\mathbb{R}^{2}$,

$$
K:=\left\{(x, y) \in \mathbb{R}^{2} ; y= \pm \varepsilon\right\} \cap \bar{B}(0,1)
$$

and take $u_{0}$ a function on the unit circle, that is equal to zero everywhere, except at one side of the tube where $u_{0}$ is equal to 1 .

\section{Figure 1.1.}

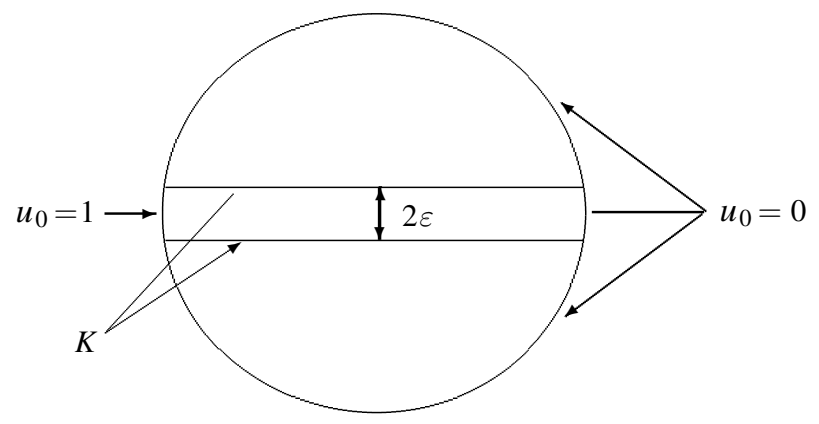

If we minimize the Dirichlet integral over all the functions that are equal to $u_{0}$ on $\partial B$, we get a linear function that goes from 1 to 0 in the tube, and which is equal to 0 everywhere else. For this minimizer $u_{\varepsilon}$ and for any fixed radius $r<1$ we have that

$$
\frac{1}{r} \int_{B(0, r)}\left|\nabla u_{\varepsilon}\right|^{2}=\int_{B(0,1)}\left|\nabla u_{\varepsilon}\right|^{2}+o(\varepsilon)
$$

thus (1.1) cannot be true for every $\varepsilon$.

Therefore, in order to guarantee that the energy decreases appropriately with the radius, we have to ensure that the set $K$ does not contain any small tubes capable to carrying energy from the outside to the inside.

The first class of sets for which the decay of energy will be true, is the class of Reifenberg-flat sets with small constant. Let us give some definitions. We denote 
by $D_{x, r}$ the normalized Hausdorff distance between two closed sets $E$ and $F$ in $B(x, r)$ defined by

$$
D_{x, r}(E, F):=\frac{1}{r}\left\{\max \left\{\sup _{y \in E \cap B(x, r)} d(y, F), \sup _{y \in F \cap B(x, r)} d(y, E)\right\}\right\} .
$$

Definition 1.1. A closed et $E \subset B$, containing the origin is said to be $\varepsilon_{0}$-Reifenbergflat in $B$ if for all $x \in E$ and for all $r$ such that $B(x, r) \subset B$ we have that

$$
\inf _{P \ni x} D_{x, r}(E, P) \leq \varepsilon_{0}
$$

where the infimum is taken over all hyperplanes $P$ that contain $x$.

Reifenberg-flat sets are introduced in [11], where a regularity theorem is stated: it is proved that every $\varepsilon_{0}$-Reifenberg-flat set in $B(0,1)$ with $\varepsilon_{0}$ small enough, is the bi-Hölder image of the unit disc.

A first result obtained in this paper is the following.

Theorem 1.2. There is a universal constant $\varepsilon_{0}<10^{-5}$ such that for all $\gamma<1$ and $0<r<\frac{1}{2}$, there is an $\varepsilon_{1}:=\varepsilon_{1}(r, \gamma)>0$ such that for every $\varepsilon_{0}$-Reifenberg-flat set $K$ in the unit ball of $\mathbb{R}^{N}$ with $P$ a hyperplane through the origin satisfying

$$
\sup \{d(y, P) ; y \in K \cap B(0,1)\} \leq \varepsilon_{1},
$$

we have that

$$
\omega_{2}(0, r) \leq r^{\gamma} \omega_{2}(0,1)
$$

for all local energy minimizers in $B(0,1) \backslash K$.

Note that we do not require (1.4) to hold for all $r$ but just that (1.4) is satisfied for a given $r$ if $\varepsilon_{1}$ is small enough, depending on $r$. However, in the case when $K$ is a $\min \left(\varepsilon_{0}, \varepsilon_{1}\right)$-Reifenberg flat set we can iterate (1.4) at every scale by applying successively infinitely many times Theorem 1.2 to get a decay for every radius $r<1$.

Actually, we will concentrate on dimension 3 because we are principally interested in a statement similar to Theorem 1.2 but for $\varepsilon_{0}$-minimal sets that will be defined below. Thus the proof will be done only in dimension 3 and with more general sets than Reifenberg-flat sets. However, one can easily see that for the case of Reifenberg-flat sets the same proof holds in any dimension, yielding Theorem 1.2 in full generality.

Before defining $\varepsilon_{0}$-minimal sets, we have to define the 3 types of minimal cones in $\mathbb{R}^{3}$. Cones of type 1 are planes in $\mathbb{R}^{3}$, also called $\mathbb{P}$. Cones of types 2 and 3 and their spines are defined as in [8] in the following way.

Definition 1.3. Define Prop $\subset \mathbb{R}^{2}$ by

$$
\begin{aligned}
\text { Prop }=\left\{\left(\mathrm{x}_{1}, \mathrm{x}_{2}\right) ; \mathrm{x}_{1} \geq 0, \mathrm{x}_{2}=0\right\} & \cup\left\{\left(x_{1}, x_{2}\right) ; x_{1} \leq 0, x_{2}=-\sqrt{3} x_{1}\right\} \\
\cup & \left\{\left(x_{1}, x_{2}\right) ; x_{1} \leq 0, x_{2}=\sqrt{3} x_{1}\right\} .
\end{aligned}
$$


Then let $Y_{0}=$ Prop $\times \mathbb{R} \subset \mathbb{R}^{3}$. The spine of $Y_{0}$ is the line $L_{0}=\left\{x_{1}=x_{2}=0\right\}$. A cone of type 2 (or of type $\mathbb{Y}$ ) is a set $Y=R\left(Y_{0}\right)$ where $R$ is the composition of a translation and a rotation. The spine of $Y$ is then the line $R\left(L_{0}\right)$. We denote by $\mathbb{Y}$ the set of all cones of type 2 .

Definition 1.4. Let $A_{1}=(1,0,0), A_{2}=\left(-\frac{1}{3}, \frac{2 \sqrt{2}}{3}, 0\right), A_{3}=\left(-\frac{1}{3},-\frac{\sqrt{2}}{3}, \frac{\sqrt{6}}{3}\right)$, and $A_{4}=\left(-\frac{1}{3},-\frac{\sqrt{2}}{3},-\frac{\sqrt{6}}{3}\right)$ be the four vertices of a regular tetrahedron centered at 0 . Let $T_{0}$ be the cone over the union of the 6 edges $\left[A_{i}, A_{j}\right] i \neq j$. The spine of $T_{0}$ is the union of the four half lines $\left[0, A_{j}\right.$ [. A cone of type 3 (or of type $\mathbb{T}$ ) is a set $T=R\left(T_{0}\right)$ where $R$ is the composition of a translation and a rotation. The spine of $T$ is the image by $R$ of the spine of $T_{0}$. We denote by $\mathbb{T}$ the set of all cones of type 3.
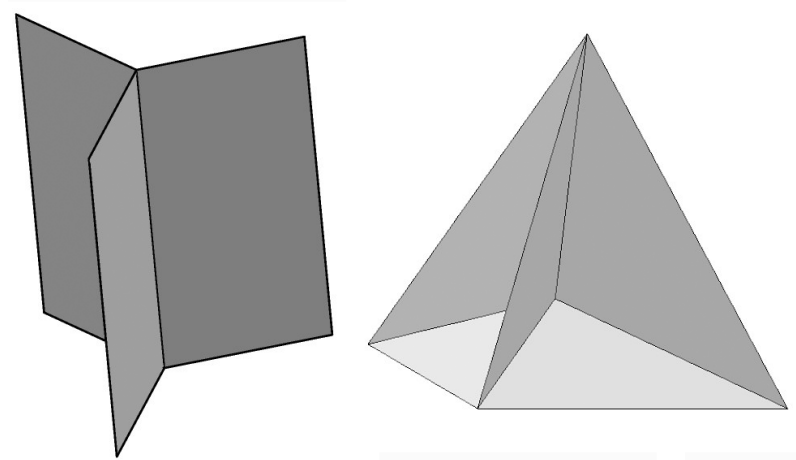

Figure 1.2. Cones $^{1}$ of type $\mathbb{Y}$ and $\mathbb{T}$.

We will also denote by type $(Z) \in\{1,2,3\}$ the number corresponding to the type of the cone $Z$.

Cones of type $\mathbb{P}, \mathbb{Y}$ and $\mathbb{T}$ are the only sets (except the empty set) in $\mathbb{R}^{3}$ that locally minimize the Hausdorff measure of dimension 2 under topological conditions (i.e. every competitor keeps the same connected components outside the competitor ball). This fact is proved in [6]. That is why cones of type $\mathbb{P}, \mathbb{Y}$ and $\mathbb{T}$ will be referred as "minimal cones". Those cones are also the blow up limits for some soap-film-like minimal surfaces in $\mathbb{R}^{3}$, which leads to the complete description of the singularities for such a surface, thanks to Jean Taylor [12].

We now give the definition of $\varepsilon_{0}$-minimal sets.

Definition 1.5. Let $B$ be a ball in $\mathbb{R}^{3}$. A closed set $E \subset B$ is said to be $\varepsilon_{0}$-minimal in $B$ if for all $x \in E$ and for all $r$ such that $B(x, r) \subset B$ we have that

$$
\inf _{Z \ni x}\left\{\frac{1}{r} \sup \{d(y, Z) ; y \in E \cap B(x, r)\}\right\} \leq \varepsilon_{0}
$$

1 Thanks to Ken Brakke for the above figures. 
where the infimum is taken over all the minimal cones of type $\mathbb{P}, \mathbb{Y}$, and $\mathbb{T}$ that contain $x$ (but are not necessarily centered at $x$ ).

Note that $\varepsilon_{0}$-minimal sets have nothing in common with minimal sets or almost minimal sets. The name "minimal" comes only from the relation with minimal cones. Moreover, in this paper we do not use the fact that the considered cones are minimal. One could prove a similar result with other cones that have good topological, flatness and hierarchy properties.

We will also find it useful to define a certain "separating condition".

Definition 1.6 (Separating). Let $Z$ be a minimal cone in $\mathbb{R}^{3}$ and $B$ a ball of radius $r$ such that $B \cap Z \neq \emptyset$. For all $a>0$ we define $Z_{a}$ by

$$
Z_{a}:=\{y \in B ; d(y, Z) \leq a\} .
$$

Let $E$ be a closed set in $B$ such that $E$ is contained in $Z_{r \varepsilon_{0}}$ for some $\varepsilon_{0}<10^{-5}$. We say that " $E$ is separating in $B$ " if the connected components of $B \backslash Z_{r \varepsilon_{0}}$ are contained in different connected components of $B \backslash E$. We denote by $\kappa^{B}$ the number of connected component of $B \backslash Z_{2 \varepsilon_{0}}$ (thus $k^{B}$ is equal to type $(Z)+1$ if $Z$ is not centered too close to $\partial B$ ).

Definition 1.5 is introduced in [8] but in a slightly different way. In [8] the inequality (1.5) is replaced by

$$
\inf _{Z \ni x} D_{x, r}(E, Z) \leq \varepsilon_{0}
$$

where $D_{x, r}$ is the normalized Hausdorff distance defined in (1.2). With this modification, $\varepsilon_{0}$-minimal sets will be called "strong $\varepsilon_{0}$-minimal sets". In our case ("weak $\varepsilon_{0}$-minimal sets") we consider the first half of the Hausdorff distance so we allow $E$ to have some holes. However, we will always suppose that our set is also separating in $B$. In general, and for technical reasons, we will always consider independently the topological separating condition and the closeness to minimal cones.

The main result on strong $\varepsilon_{0}$-minimal sets is [8, Theorem 2.1] which says that for $\varepsilon_{0}$ small enough, every strong $\varepsilon_{0}$-minimal set is locally the bi-Hölder image of a minimal cone. This is a generalization of Reifenberg's topological disc theorem that was mentioned above. So $\varepsilon_{0}$-minimal sets can be seen as a generalization of Reifenberg-flat sets.

In particular, a strong $\varepsilon_{0}$-minimal set is a separating set. This is a consequence of [8] but one can also simply prove it from the definitions. A consequence is that a strong $\varepsilon_{0}$-minimal set in $B$ is a weak $\varepsilon_{0}$-minimal set that separates in $B$. This is why our result, which will be stated later for weak $\varepsilon_{0}$-minimal sets with the separating condition, applies directly to strong $\varepsilon_{0}$-minimal sets in $B$. As a result, it applies to $\varepsilon_{0}$-Reifenberg-flat sets (hence Theorem 1.2 follows) and we also have

Theorem 1.7. There is a universal constant $\varepsilon_{0}<10^{-5}$ such that for all $\gamma<1$ and $0<r<\frac{1}{2}$, there is an $\varepsilon_{1}:=\varepsilon_{1}(r, \gamma)>0$ such that for every strong $\varepsilon_{0}$-minimal set $K$ in the unit ball of $\mathbb{R}^{3}$ with $Z^{0}$ a minimal cone satisfying

$$
\sup \left\{d\left(x, Z^{0}\right) ; x \in K \cap B(0,1)\right\} \leq \varepsilon_{1},
$$


we have that

$$
\omega_{2}(0, r) \leq r^{\gamma} \omega_{2}(0,1)
$$

for all local energy minimizers in $B(0,1) \backslash K$.

Let us now introduce the unilateral $\beta$-number associated to minimal cones, for a closed set $E \subset B$ :

$$
\beta(x, r):=\inf _{Z \ni x}\left\{\frac{1}{r} \sup \{d(y, Z) ; y \in E \cap B(x, r)\}\right\}
$$

where the infimum is taken over all the minimal cones $Z$ of type $\mathbb{P}, \mathbb{Y}$ or $\mathbb{T}$ that contain $x$. We will also use the following definition.

Definition 1.8. Let $F:=\left\{B_{i}\right\}_{i \in I}$ be a family of balls in $\mathbb{R}^{N}$. We say that $F$ is in bounded cover, with constant $C_{0}$, if for all $x \in \mathbb{R}^{N}$,

$$
\sharp\left\{i \in I ; x \in B_{i}\right\} \leq C_{0} .
$$

We are now ready to define $\left(\varepsilon_{0}, \varepsilon\right)$-minimal sets, and state the main theorem that will in particular imply Theorem 1.2 and Theorem 1.7.

Definition 1.9. Let $E \subset \bar{B}(0,1) \subset \mathbb{R}^{3}$ be a closed set with locally finite $\mathcal{H}^{2}$ measure. Let $\varepsilon$ and $\varepsilon_{0}$ be two positive constants such that $0<\varepsilon \leq \varepsilon_{0}<10^{-5}$. We say that $E$ is $\left(\varepsilon_{0}, \varepsilon\right)$-minimal if there is a constant $C_{0}$ and a family of balls $\left\{B_{i}\right\}_{i \in I}:=\left\{B\left(x_{i}, r_{i}\right)\right\}_{i \in I}$ such that $\left\{2 B_{i}\right\}$ is in bounded cover with constant $C_{0}$, centered on $E$ (but not necessarily contained in $B(0,1)$ ), and such that:

i) $\forall i \in I ; r_{i} \leq \varepsilon$.

ii) $E \backslash \bigcup_{i \in I} B\left(x_{i}, r_{i}\right)$ is $\varepsilon_{0}$-minimal in $B(0,1)$.

iii) There is a minimal cone $Z$ centered at the origin such that

$$
E \subset\{y \in B(0,2) ; d(y, Z) \leq \varepsilon\} .
$$

iv) For all $i \in I$ and for all $r>r_{i}$ with $B\left(x_{i}, r\right) \subset B(0,1)$, we have $\beta\left(x_{i}, r\right) \leq \varepsilon_{0}$.

v) $E$ is separating in $B(0,1)$.

The separating condition in $v$ ) uses the cone of iii).

One can see an $\left(\varepsilon_{0}, \varepsilon\right)$-minimal set as an $\varepsilon_{0}$-minimal set in $B(0,1)$ except in a collection of tiny bad balls $B_{i}$ of radius less than $\varepsilon$. Note that $E \cap B(0,1) \backslash \bigcup_{i \in I} B_{i}$ could be empty. This means that any closed set satisfying iii) and $v)$ is an $\left(\varepsilon_{0}, \frac{\varepsilon}{\varepsilon_{0}}\right)$ minimal set with for instance $\left\{B_{i}\right\}$ a Vitali subfamily of $\left\{B\left(x, \frac{\varepsilon}{\varepsilon_{0}}\right)\right\}_{x \in E \cap B(0,1)}$. On the other hand, if $E$ is an $\varepsilon_{0}$-minimal set then it is $\left(\varepsilon_{0}, \varepsilon_{0}\right)$-minimal with an empty family of balls $\left\{B_{i}\right\}$. However, the more interesting case will be the intermediate situation, when the family $\left\{B_{i}\right\}$ is neither empty nor too scattered.

It is worth mentioning that our theorem will show a decay of energy for local energy minimizers in the complement of $E^{\rho}$ and not $E$, where $E^{\rho}$ is a new set obtained by adding a wall of size $\varepsilon$ consisting of the union of all the $\partial B_{i}$ that meet a 
fixed "tube-cutting sphere" $\partial B(0, \rho)$. Therefore, the more bad balls $B_{i}$ 's there are, the more wall we have to build in order to cut the little tubes and to make the result true.

In general, $\left(\varepsilon_{0}, \varepsilon\right)$-minimal sets can be obtained by a stopping time argument. Indeed, let $\varepsilon<\varepsilon_{0}$ be some small constants and consider a closed set $E \subset \bar{B}(0,1)$ such that

$$
\sup \{d(y, Z) ; y \in E \cap B(0,1)\} \leq \varepsilon_{0} \varepsilon
$$

for a minimal cone $Z$ centered at the origin and assume that $E$ is also separating in $B(0,1)$. Then for all $x \in E \cap B(0,1)$, define the stopping time

$$
d(x):=\inf \left\{r ; \forall t \in[r, d(x, \partial B(0,1))], \beta(x, t) \leq \varepsilon_{0}\right\} .
$$

Observe that (1.8) implies $d(x) \leq \varepsilon$. Then if $\left\{B_{i}\right\}_{i \in I}$ is a Vitali subfamily of the balls

$$
\{B(x, d(x))\}_{x \in K \cap B(0,1)},
$$

one have that $E$ is an $\left(\varepsilon_{0}, \varepsilon\right)$-minimal set. This will be used in a second paper to prove the regularity for the singular set of a minimizer for the Mumford-Shah functional in $\mathbb{R}^{3}$ near minimal cones (see [9]). The latter is the main motivation for introducing $\left(\varepsilon_{0}, \varepsilon\right)$-minimal sets but we hope that the stopping time technique described above could be useful for other free boundary sets that are coming from other minimization problems.

So we want to prove a decay of normalized energy in the complement of $\left(\varepsilon_{0}, \varepsilon\right)$-minimal sets. As was mentioned previously, this type of set is too general to hope to obtain such a result since $\left(\varepsilon_{0}, \varepsilon\right)$-minimal sets allow the existence of little tubes hidden in the bad balls $B_{i}$ and that could carry some energy from exterior to interior. This is why we consider a "tube-cutting sphere" of a certain radius $\rho$ that is not fixed but just belongs to $\left[\frac{1}{2}, \frac{3}{4}\right]$. Recall that we denote by $B:=B(0,1)$ the unit ball.

For all $i \in I$ we let

$$
B_{i}^{\prime}:=C_{1} B_{i}=B\left(x_{i}, C_{1} r_{i}\right)
$$

where $C_{1}>1$ is a constant that will be chosen later, depending on $C_{0}$ and other geometric constants.

For all $\rho \in\left[\frac{1}{2}, \frac{3}{4}\right]$ and for every $\left(\varepsilon_{0}, \varepsilon\right)$-minimal set $E$ we define

$$
I_{\rho}:=\left\{i \in I ; B_{i} \cap \partial B(0, \rho) \neq \emptyset\right\}
$$

and

$$
E^{\rho}:=\left(E \backslash \bigcup_{i \in I_{\rho}} B_{i}^{\prime}\right) \cup \bigcup_{i \in I_{\rho}} \partial B_{i}^{\prime} .
$$

Observe that since $E^{\rho}$ is closed and $\partial B \backslash E^{\rho}$ is locally a $C^{1}$ graph, any

$$
u \in W^{1,2}\left(B \backslash E^{\rho}\right)
$$


has a trace on $\partial B \backslash E^{\rho}$ defined by taking some radial limits (see [7, Chapter 13]). Thus we can define

$$
\mathcal{F}\left(E^{\rho}, u\right):=\left\{v \in W^{1,2}\left(B \backslash E^{\rho}\right) ; v=u \quad \mathcal{H}^{2} \text { - a.e. on } \partial B \backslash E^{\rho}\right\}
$$

and consider the problem

$$
\min _{v \in \mathcal{F}\left(E^{\rho}, u\right)} \int_{B \backslash E^{\rho}}|\nabla v(x)|^{2} d x .
$$

Finally set

$$
\begin{aligned}
& U\left(E^{\rho}\right):=\left\{u /\|\nabla u\|_{L^{2}\left(B \backslash E^{\rho}\right)} ; u \in W^{1,2}\left(B \backslash E^{\rho}\right),\|\nabla u\|_{2}>0\right. \\
& \text { and } u \text { is a solution for the Problem (1.10) }\} .
\end{aligned}
$$

Note that if $\varepsilon$ is smaller than $1 / 4 C_{1}$, all the $B_{i}^{\prime}$ for $i \in I_{\rho}$ do not meet $\partial B$ so all the functions in $U\left(E^{\rho}\right)$ are constant in each $B_{i}^{\prime}$ for $i \in I_{\rho}$.

Let $\varepsilon_{0}<10^{-5}$ be fixed. For all $\varepsilon<\frac{1}{4}$ we introduce

$$
\begin{aligned}
& \Lambda(\varepsilon):=\{(u, E, \rho) \text { that verify }(*)\} \\
& (*) \begin{cases}E & \text { is }\left(\varepsilon_{0}, \varepsilon\right) \text {-minimal } \\
\rho & \in\left[\frac{1}{2}, \frac{3}{4}\right] \\
u & \in U\left(E^{\rho}\right) .\end{cases}
\end{aligned}
$$

For $i \in\{1,2,3\}$ we denote by $\Lambda_{i}(\varepsilon)$ the elements of $\Lambda(\varepsilon)$ such that the cone $Z$ of condition iii) of Definition 1.9 is of type $i$.

For all $(u, E, \rho) \in \Lambda(\varepsilon)$ and all $0<r<1$, recall that

$$
\omega_{2}(0, r):=\frac{1}{r^{2}} \int_{B(0, r) \backslash E^{\rho}}|\nabla u(x)|^{2} d x .
$$

We now come to the main result.

Theorem 1.10. There is a universal constant $\varepsilon_{0}<10^{-5}$ such that for all $i \in$ $\{1,2,3\}, \gamma<2(\sqrt{2}-1)$ and $0<r<\frac{1}{2}$, there is an $\varepsilon_{1}:=\varepsilon_{1}(r, \gamma)>0$ such that for all $(u, E, \rho) \in \Lambda_{i}\left(\varepsilon_{1}\right)$ we have

$$
\omega_{2}(0, r) \leq r^{\gamma} .
$$

Theorem 1.10 is proved by contradiction and compactness. So the first step is to prove a decay estimate on the energy when $K$ is a minimal cone. This is done in Section 2. In fact we will prove that in this case the normalized energy increases like a power of radius. To show this, we use an argument that A. Bonnet used in dimension 2, that link the decay of normalized energy and the spectrum of the 
spherical Laplacian on $\partial B(0, r) \backslash K$. In particular we give a lower bound for the first eigenvalue in those domains.

Section 3 and Section 4 are devoted to a general method to obtain extensions of functions $u$ near $K$ by a Whitney type construction. This work gives some useful competitors for energy minimizing functions in the complement of $\left(\varepsilon_{0}, \varepsilon\right)$-minimal sets. This approach might be interesting for other problems. In particular it will be used twice in the forthcoming paper [9].

Finally in Section 5, we give the proof of Theorem 1.10. The argument is inspired by what L. Ambrosio, N. Fusco, and D. Pallara did in their theorem concerning the decay of energy in [1] (see also in [2, Theorem 8.19]). The difficulty here, and also the key ingredient of the proof, is to estimate the energy close to the set $E$ (inequality (5.4)). In their case, L. Ambrosio, N. Fusco, and D. Pallara obtained this estimate by approximating the set $E$ with a Lipschitz surface and by controlling the difference with the "Tilt estimate" which is a typical tool for the flat case. Here, we shall use the Whitney extension from the preceding sections to show a similar estimate which is independent from the geometric situation like when the set is close to a cone $\mathbb{Y}$ or a cone $\mathbb{T}$, where the Tilt there is difficult to control.

\section{Monotonicity in the complement of a minimal cone}

We want to prove first that if $K=Z$ with $Z$ a cone of type $\mathbb{Y}$ or $\mathbb{T}$ centered at the origin and $u$ locally minimizes the energy in $B(0,1) \backslash Z$ then

$$
\omega_{2}(0, r) \leq r^{2(\sqrt{2}-1)} \omega_{2}(0,1) \quad \forall r<1 .
$$

To prove (2.1), we will adapt an argument that A. Bonnet [3] used in dimension 2, and to do this, we will need the following Proposition.

Proposition 2.1. Let $Z$ be a minimal cone in $\mathbb{R}^{3}$ centered at 0 and let $\Omega_{r}$ be a connected component of $\partial B(0, r) \backslash Z$. Then for all functions $f \in W^{1,2}\left(\Omega_{r}\right)$ we have

$$
\int_{\Omega_{r}}\left|f-m_{f}\right|^{2} d w \leq \frac{1}{2} r^{2} \int_{\Omega_{r}}\left|\nabla_{\tau} f\right|^{2} d w .
$$

Proof. Let $\lambda_{1}$ be the first positive eigenvalue for $-\Delta_{S}$ (spherical Laplacian) in $\Omega_{1}$ with Neumann condition on the boundary of $\Omega_{1}$. Then we have

$$
\int_{\Omega_{r}}\left|f-m_{f}\right|^{2} d w \leq \frac{1}{\lambda_{1}} r^{2} \int_{\Omega_{r}}\left|\nabla_{\tau} f\right|^{2} d w .
$$

It is well known that if $Z$ if of type $\mathbb{P}$, then $\lambda_{1}=2$ (in dimension $N \geq 2$ we have $\lambda_{1}=N-1$ see for instance of [7, Exercice 21, page 538]).

If $Z$ is of type $\mathbb{Y}$, then by of [5, Lemma 4.1] applied with $\omega=\frac{2 \pi}{3}$ and with Neumann boundary conditions, we get $\lambda_{1}=2$ and (2.2) follows. 
So we have to consider the case when $Z$ is of type $\mathbb{T}$. Let $f$ be an eigenfunction for the first positive Neumann eigenvalue. For $1 \leq i \leq 3$ we denote by $\delta_{i}$ the three symmetry axis of $\Omega_{1}$ and we denote by $s_{i}$ the corresponding symmetries. If $f$ is symmetric with respect to all the three axis, then by reflection we could extend $f$ to the entire sphere for which it is well known that the first eigenvalue is equal to 2 .

If $f$ is not symmetric by the three axis, then there is one, for instance $\delta_{1}$, such that $f$ is not symmetric. Then we consider the anti-symmetric function

$$
g=f-f \circ s_{1} \text {. }
$$

By this way, $g$ is still an eigenfunction associated to $\lambda_{1}$. Moreover, we have that $g$ vanishes on $\delta_{1}$. The axis $\delta_{1}$ cut $\Omega_{1}$ in two isometric triangles. We call $\Omega^{\prime}$ one of them. Now we consider $\Sigma$ a connected component of $S^{2} \backslash Y$ where $Y$ is of type $\mathbb{Y}$ such that $\Sigma$ contains $\Omega$. $\Sigma$ is also cut by $\delta_{1}$ and we denote by $\Sigma^{\prime}$ the connected component that contains $\Omega^{\prime}$. Now we apply of [5, Proposition 4.3] with $G=\Omega^{\prime}$, $\partial_{D} G=\delta_{1} \cap \partial \Omega^{\prime}, \partial_{N} G=\partial \Omega^{\prime} \backslash \delta_{1}, G^{\prime}=\Sigma^{\prime}, \partial_{N} G^{\prime}=\partial \Sigma^{\prime} \backslash \delta_{1}$ and $\partial_{D} G^{\prime}=\delta_{1} \cap \partial \Sigma^{\prime}$. We obtain that

$$
\lambda_{1} \geq \mu\left(\Sigma^{\prime}\right)
$$

where $\mu\left(\Sigma^{\prime}\right)$ is the first positive eigenvalue in $\Sigma^{\prime}$ with Neumann condition on $\partial \Sigma^{\prime} \backslash \delta_{1}$ and Dirichlet condition on $\delta_{1} \cap \partial \Sigma^{\prime}$. Now applying of [5, Lemma 4.1] again with $\omega=\frac{2 \pi}{3}$, but now with mixed boundary conditions, we get $\mu\left(\Omega^{\prime}\right)=2$, thus

$$
\lambda_{1} \geq 2
$$

and the lemma follows.

Lemma 2.2. Let $Z$ be a minimal cone in $\mathbb{R}^{3}$ centered at 0 . Then for all local energy minimizer $u$ in $B(0,1) \backslash Z$ and for all $a, r<1$ we have

$$
\omega_{2}(0, a r) \leq a^{2(\sqrt{2}-1)} \omega_{2}(0, r)
$$

Moreover, $r \mapsto \omega_{2}(0, r)$ is increasing.

Proof. Set

$$
E(r)=\int_{B(0, r) \backslash Z}|\nabla u|^{2} .
$$

For almost every $r$ the derivative of $r \mapsto E(r)$ exists,

$$
E^{\prime}(r)=\int_{\partial B(0, r) \backslash Z}|\nabla u|^{2} \quad \text { and } \quad E(r)=\int_{0}^{r} E^{\prime}(t) d t
$$

(see [7, Lemma 47.4, page 316]). 
We want to prove an inequality of type

$$
E(r) \leq \operatorname{Cr} E^{\prime}(r)
$$

where $C$ is a contant that will be explicited later.

Firstly, since $u$ is a harmonic function and since $\frac{\partial}{\partial n} u=0$ on $Z$ (where $Z$ is regular), an integration by parts gives

$$
E(r)=\sum_{j=1}^{J} \int_{S_{j}} u \frac{\partial u}{\partial r}
$$

with $S_{j}$ the connected components of $\partial B(0, r) \backslash Z$. To justify the integration by parts in this "regular polyhedral domain", one can find a lemma in [10, (Lemma 1.6.)] and also in [4, Theorem 22.6]. In addition, one might prove (2.3) without integrating by parts but just by using that $u$ is an energy minimizing function as in [7, page 320], but the author didn't verify.

Denote by $A_{j}$ the connected components of $B(0, r) \backslash Z$ which boundary contains $S_{j}$. An other integration by parts in $S_{j}$ gives

$$
\int_{S_{j}} \frac{\partial u}{\partial r}=\int_{A_{j}} \Delta u=0
$$

Thus we can subtract by a constant and we find

$$
\begin{aligned}
\int_{S_{j}} u \frac{\partial u}{\partial r} & =\int_{S_{j}}\left[u-c_{j}(u)\right] \frac{\partial u}{\partial r} \\
& \leq\left[\int_{S_{j}}\left[u-c_{j}(u)\right]^{2}\right]^{\frac{1}{2}}\left[\int_{S_{j}}\left(\frac{\partial u}{\partial r}\right)^{2}\right]^{\frac{1}{2}} .
\end{aligned}
$$

Then by use of $a b \leq \frac{1}{2}\left[\lambda^{-1} a^{2}+\lambda b^{2}\right]$ with $\lambda$ a positive constant to be chosen later,

$$
\begin{aligned}
\int_{S_{j}} u \frac{\partial u}{\partial r} & \leq \frac{1}{2 \lambda}\left[\int_{S_{j}}\left[u-c_{j}(u)\right]^{2}\right]+\frac{\lambda}{2} \int_{S_{j}}\left(\frac{\partial u}{\partial r}\right)^{2} \\
& \leq \frac{1}{4 \lambda} r^{2} \int_{S_{j}}\left|\nabla_{\tau} u\right|^{2}+\frac{\lambda}{2} \int_{S_{j}}\left(\frac{\partial u}{\partial r}\right)^{2}
\end{aligned}
$$

then by setting $\lambda=\frac{r}{\sqrt{2}}$,

$$
\int_{S_{j}} u \frac{\partial u}{\partial r} \leq \frac{1}{2 \sqrt{2}} r \int_{S_{j}}|\nabla u|^{2} .
$$

Finally, summing over $j$,

$$
E(r) \leq \frac{1}{2 \sqrt{2}} r E^{\prime}(r)
$$


This estimate shows that the derivative of $\omega_{2}(0, r)$ is positive, in other words $r \mapsto$ $\omega_{2}(0, r)$ is increasing. To have now the estimate about the speed of increasing, consider $g(r):=\ln (E(r))$. By absolute continuity we have

$$
g(r)-g(a r)=\int_{a r}^{r} \frac{E^{\prime}(t)}{E(t)} d t \geq \int_{a r}^{r} \frac{2 \sqrt{2}}{t} d t \geq 2 \sqrt{2} \ln \left(\frac{1}{a}\right) .
$$

Hence

$$
E(a r) \leq a^{2 \sqrt{2}} E(r)
$$

Then we divide by $(a r)^{2}$

$$
\frac{1}{(a r)^{2}} E(a r) \leq a^{2(\sqrt{2}-1)} \frac{1}{r^{2}} E(r)
$$

and this implies the lemma.

\section{Some geometric lemmas}

Before doing the Whitney extension, we have to discuss some geometric facts. We begin this section with a lemma that will allow us to work with "almost centered cones". Let us first say a word about what we call the center of a minimal cone. By convention, a cone of type $\mathbb{P}$ admits any of its own points as center. For cones of type $\mathbb{Y}$, any point on the spine is a center. Finally, a cone of type $\mathbb{T}$ admits only one possible center defined by the intersection of its 4 edges.

\subsection{The recentering lemma}

Lemma 3.1 (Recentering). Let $Z$ be a minimal cone in $\mathbb{R}^{3}$ that contains 0 (but is not necessarily centered at 0 ). Then for all $r_{0}>0$ and for all constant $V \geq 1$ there is a $r_{1}$ such that

$$
r_{1} \in\left\{r_{0}, V r_{0}, V^{2} r_{0}\right\}
$$

and such that we can find a cone $Z^{\prime}$, containing 0 and centered in $B\left(0, \frac{1}{V} r_{1}\right)$ with $Z \cap B\left(0, r_{1}\right)=Z^{\prime} \cap B\left(0, r_{1}\right)$.

Proof. Let us first consider the situation in the ball $B\left(0, r_{0}\right)$. If type $(Z)=1$ then $Z$ is a plane that contains $x$, thus $x$ is a center and we can take $r_{1}=r_{0}$.

Suppose now that $Z$ is of type $\mathbb{Y}$ and assume that all the points on the spine of $Z$ are in $\mathbb{R}^{3} \backslash B\left(0, \frac{1}{V} r_{0}\right)$ (otherwise we could take $r_{1}=r_{0}$ that is case 1 of Figure 3.1 ). If there is no point on the spine of $Z$ in $B\left(0, r_{0}\right)$, we can take $Z^{\prime}$ a plane that is equal to $Z$ in $B\left(0, r_{0}\right)$. Otherwise we are in case 2 of Figure 3.1 and we can take $r_{1}=V r_{0}$.

Now it remains to consider the case when $Z$ is of type $\mathbb{T}$. We discuss it in the same way. If the center of $Z$ is not in $B\left(0, r_{0}\right)$, and if there is no point on the spine of a cone of type $\mathbb{Y}$ in $B\left(0, r_{0}\right)$, then we take $r_{1}=r_{0}$ and for $Z^{\prime}$ we take a plane. 


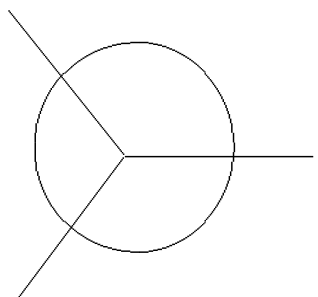

Case 1

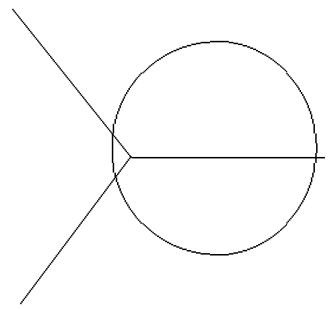

Case 2

\section{Figure 3.1.}

Now if there is some point of type $\mathbb{Y}$ in $B\left(0, r_{0}\right)$ but no point of type $\mathbb{T}$, and if in addition there is a $\mathbb{Y}$ spine passing trough $B\left(0, \frac{1}{V} r_{0}\right)$ we can take for $Z^{\prime}$ a cone of type $\mathbb{Y}$ and fix $r_{1}=r_{0}$. Now if there is some point of type $\mathbb{Y}$ in $B\left(0, r_{0}\right) \backslash B\left(0, \frac{1}{V} r_{0}\right)$, we try $r_{1}=V r_{0}$. If there is no point of type $\mathbb{T}$ in $B\left(0, V r_{0}\right)$, we can take for $Z^{\prime}$ a cone of type $\mathbb{Y}$ and $r_{1}=V r_{0}$ (case 1 of Figure 3.2). Otherwise we take for $Z^{\prime}$ a cone of type $\mathbb{T}$ and we fix $r_{1}=V^{2} r_{0}$.

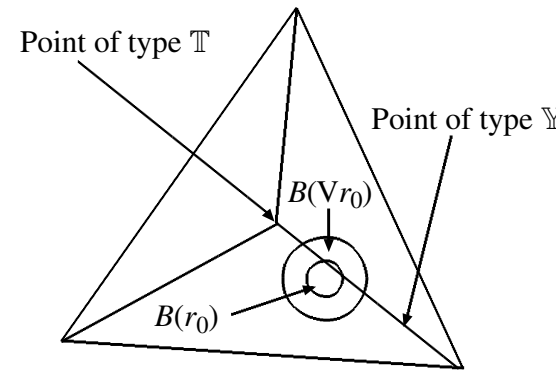

Case 1

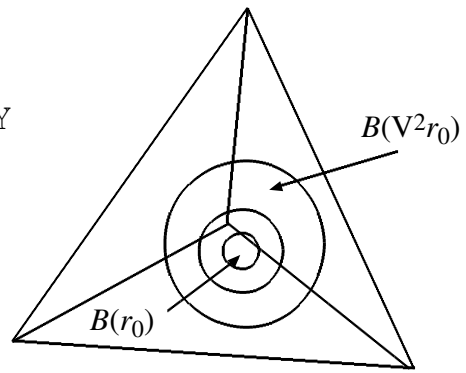

Case 2

\section{Figure 3.2.}

Finally if $Z$ is of type $\mathbb{T}$ and its center lies in $B\left(0, r_{0}\right)$, then if the center lies in $B\left(0, \frac{1}{V} r_{0}\right)$ we take $r_{0}=r_{1}$ and $Z=Z^{\prime}$, otherwise we take $r_{1}=V r_{0}$ and $Z^{\prime}=Z$.

Definition 3.2 (Almost centered). Let $Z$ be a minimal cone and $B$ a ball that meets $Z$. We say that $Z$ is almost centered with constant $V$ if the center of $Z$ lies in $\frac{1}{V} B$. If $V=2$ we just say that $Z$ is almost centered in $B$.

\subsection{The geometric function and general assumptions}

Here we describe the general situation that will appear in next sections. Let $K$ be a closed set in $\bar{B}\left(x_{0}, r_{0}\right)$ such that $\mathcal{H}^{2}\left(K \cap \bar{B}\left(x_{0}, r_{0}\right)\right)<+\infty$. Suppose that there is a positive constant $\varepsilon_{0}<10^{-5}$ and a minimal cone $Z$, centered at $x_{0}$, such that

$$
\sup \left\{d(x, Z) ; x \in K \cap B\left(x_{0}, r_{0}\right)\right\} \leq r_{0} \varepsilon_{0}
$$


and that $K$ is separating in $B\left(x_{0}, r_{0}\right)$. For all $x \in K \cap B\left(x_{0}, r_{0}\right)$ and $r>0$ such that $B(x, r) \subset B\left(0, r_{0}\right)$ recall that

$$
\beta(x, r)=\inf _{Z \ni x} \frac{1}{r} \sup \{d(x, Z) ; x \in K \cap B(x, r)\} .
$$

Let $\rho \in\left[\frac{1}{2} r_{0}, \frac{3}{4} r_{0}\right]$ and assume that we have an application

$$
\delta: B\left(x_{0}, \rho\right) \rightarrow\left[0, \frac{1}{4} r_{0}\right]
$$

with the property that

$$
\beta(x, r) \leq \varepsilon_{0}, \text { for all } x \in K \cap B\left(x_{0}, \rho\right) \text { and } r \text { such that } \delta(x) \leq r \leq \frac{1}{4} r_{0} .
$$

In addition we suppose that

$$
\delta \text { is } C_{0} \text { - Lipschitz. }
$$

The application $\delta$ will be called the "geometric function".

Definition 3.3 (Hypothesis $H 1$ ). We will say that a closed set $K \subset B\left(x_{0}, r_{0}\right)$ with finite $\mathcal{H}^{2}$ measure is satisfying hypothesis $H 1$ if

i) There is a minimal cone $Z$ centered at $x_{0}$ that verify (3.1) for a "geometric constant" $\varepsilon_{0}<10^{-5}$.

ii) $K$ is separating in $B\left(x_{0}, r_{0}\right)$.

iii) There is a geometric function $\delta$ satisfying (3.2), (3.3) and (3.4) for a radius $\rho \in\left[\frac{1}{2} r_{0}, \frac{3}{4} r_{0}\right]$ and a Lipschitz constant $C_{0}$.

For example, if we have $\beta\left(x_{0}, r_{0}\right) \leq \varepsilon$ with $\varepsilon<\frac{1}{4} \varepsilon_{0}$, and if in addition $K$ is separating, then we have Hypothesis $H 1$ with $\delta(x)=\frac{\varepsilon}{\varepsilon_{0}} r_{0}$ everywhere in $B\left(x_{0}, \rho\right)$.

An other example is given by a Reifenberg-flat set $K$ in $B\left(x_{0}, 2 r_{0}\right)$, containing $x_{0}$ and with constant $\varepsilon_{0}$ less than $10^{-6}$. Then we have Hypothesis $H 1$ on $K$ with $\delta=0$ everywhere.

Recall that if $E$ is separating in $B(x, r)$ associated to a cone $Z$ centered at $x$, we denote by $\kappa^{B(x, r)}$ the number of connected components of $B(x, r) \backslash Z$ (see Definition 1.6).

Under Hypothesis $H 1$ we will always denote by $A_{k}\left(x_{0}, r_{0}\right)$ for $k \in \mathbb{N} \cap$ $\left[1, \kappa^{B\left(x_{0}, r_{0}\right)}\right]$ the connected components of

$$
B\left(x_{0}, r_{0}\right) \backslash\left\{y \in B(x, r) ; d(y, Z) \leq \varepsilon_{0} r_{0}\right\}
$$

and we will call $\Omega_{k}\left(x_{0}, r_{0}\right)$ the connected component of $B\left(x_{0}, r_{0}\right) \backslash K$ that contains $A_{k}\left(x_{0}, r_{0}\right)$. 


\subsection{The orientation lemma}

Now we have to discuss orientation and separation.

Lemma 3.4 (Orientation). Let $K$ be a closed set in $B\left(x_{0}, r_{0}\right)$ satisfying Hypothesis $H 1$ with a geometric function $\delta$, a minimal cone $Z$, a radius $\rho$ and a constant $\varepsilon_{0}<10^{-5}$. Let $B(x, r)$ be a ball contained in $B\left(x_{0}, r_{0}\right)$ such that $x \in K \cap B\left(x_{0}, \rho\right)$ and

$$
\delta(x) \leq r \leq \frac{r_{0}}{32} .
$$

Let $r_{1}$ be the radius (equal to $r, 2 r$ or $4 r$ ) such that $Z\left(x, r_{1}\right)$ is almost centered in $B\left(x, r_{1}\right)$ where $Z\left(x, r_{1}\right)$ denotes the minimal cone $\varepsilon_{0}$-close to $K$ in $B\left(x, r_{1}\right)$. We also call $A_{k}\left(x, r_{1}\right), k \in \mathbb{N} \cap\left[1, \kappa^{B\left(x, r_{1}\right)}\right]$, the connected components of

$$
\left\{y \in B\left(x, r_{1}\right) ; d\left(y, Z\left(x, r_{1}\right)\right) \geq \varepsilon_{0} r_{1}\right\} .
$$

Then $B\left(x, r_{1}\right)$ is well oriented in $B\left(x_{0}, r_{0}\right)$, i.e. $B\left(x, r_{1}\right)$ verifies the two following points:

i) $K$ is separating in $B\left(x, r_{1}\right)$.

ii) There is an injective application

$$
l: \mathbb{N} \cap\left[1, \mathcal{K}^{B\left(x, r_{1}\right)}\right] \rightarrow \mathbb{N} \cap\left[1, \mathcal{K}^{B\left(x_{0}, r_{0}\right)}\right]
$$

such that $A_{k}\left(x, r_{1}\right) \subset \Omega_{l(k)}\left(x_{0}, r_{0}\right)$.

Proof. We consider the balls $B^{p}:=B\left(x, 2^{p} r_{1}\right)$ for $p \in \mathbb{N} \cap[0, P]$ where $P$ is such that

$$
\frac{1}{16} r_{0} \leq 2^{P} r_{1} \leq \frac{1}{8} r_{0}
$$

It is always possible because $r_{1} \leq \frac{1}{8} r_{0}$. Therefore, every $B^{p}$ is contained in $B\left(x_{0}, r_{0}\right)$. We define also $B^{P+1}:=B\left(x_{0}, r_{0}\right)$ and $Z^{p}$ is the minimal cone that is $\varepsilon_{0}$-close to $K$ in $B^{p}$ (we know that there is one for all $p$ because the radius of $B^{p}$ is larger than $\delta(x)$ ). Now we use Lemma 3.1 to extract among the $B^{p}$ a subsequence $B^{\sigma(p)}$ such that for all $p, B^{\sigma(p)}$ is almost centered and such that the radius of each ball is not larger than eight times the radius of the preceding one. We still denote by $B^{p}$ this subsequence (instead of $\left.B^{\sigma(p)}\right) . B^{0}=B\left(x, r_{1}\right)$ is the beginning ball and $B^{P+1}$ is $B\left(x_{0}, r_{0}\right)$. Nevertheless, the radius of $B^{p}$ is not exactly $2^{p} r_{0}$ but equivalent with a factor 4 .

Now we are going to prove by induction that $B^{p}$ is well oriented for all $p$.

Hypothesis $H 1$ in $B\left(x_{0}, r_{0}\right)$ clearly shows that $B^{P+1}:=B\left(x_{0}, r_{0}\right)$ is well oriented. Next we consider $p \leq P$. We have to show that if $B^{p+1}$ is well oriented, then $B^{p}$ is well oriented. We denote by $\kappa^{p}$ instead of $\kappa^{B^{p}}$ the number of connected components and we denote by $r_{p}$ the radius of $B^{p}$. We know that

$$
K \cap B^{p} \subset Z_{r_{p} \varepsilon_{0}}^{p}:=\left\{y ; d\left(y, Z^{p}\right) \leq r_{p} \varepsilon_{0}\right\}
$$


and in addition $K$ is separating in $B^{p}$. Let $A_{k}^{p}$ be for $k \in \mathbb{N} \cap\left[1, \kappa^{p}\right]$ the connected components of $B^{p} \backslash Z_{r_{p} \varepsilon_{0}}^{p}$.

Now consider $B^{P}$. Firstly note that $K$ is separating in $B^{p}$. To see this we have to show that connected components of $B^{p} \backslash Z_{r_{p} \varepsilon_{0}}^{p}$ are in different connected components of $B^{p} \backslash K$. Since

$$
K \cap B^{p} \subset Z_{r_{p} \varepsilon_{0}}^{p}
$$

and $\varepsilon_{0}<10^{-5}$, we can choose for all $k$ a point $a_{k}^{p}$ such that $a_{k}^{p} \in A_{k}^{p}$, and $d\left(a_{k}^{p}, K\right) \geq \frac{r_{p}}{10}$ (because the cones $Z^{p}$ are almost centered).

Since $d\left(a_{k}^{p}, K\right) \geq \frac{r_{p}}{10}$, then $d\left(a_{k}^{p}, Z_{r_{p} \varepsilon_{0}}^{p}\right) \geq r_{p}\left(\frac{1}{10}-\varepsilon_{0}\right)$ and by use of (3.5) we can deduce that $d\left(a_{k}^{p}, Z_{r_{p+1} \varepsilon_{0}}^{p+1}\right) \geq r_{p+1}\left(\frac{1}{10}-10 \varepsilon_{0}\right)$. It follows that for $k \in$ $\mathbb{N} \cap\left[1, \kappa^{p}\right]$ there is an $l \in \mathbb{N} \cap\left[1, \kappa^{p+1}\right]$ such that $a_{k}^{p} \in A_{l}^{p+1}$.

In addition,

$$
\text { the } a_{k}^{p} \text { are in different connected components of } B^{p+1} \backslash Z_{\varepsilon_{0} r_{p+1}}^{p+1} \text {. }
$$

Indeed, suppose that there is $k_{1}$ and $k_{2}$ such that $a_{k_{1}}^{p}$ and $a_{k_{2}}^{p}$ are both in the same connected component of $B^{p+1} \backslash Z_{\varepsilon_{0} r_{p+1}}^{p+1}$. Therefore, since $d\left(a_{k}^{p}, Z_{r_{p+1} \varepsilon_{0}}^{p+1}\right) \geq r_{p+1} \frac{1}{11}$ and since the $Z^{p}$ are almost centered, we can deduce that there is a continuous path $\Gamma$ from $a_{k_{1}}^{p}$ to $a_{k_{2}}^{p}$ such that all the points of $\Gamma$ are situated at a distance larger than $\frac{1}{11} r_{p+1}$ from $Z^{p+1}$, consequently larger than $\frac{1}{100} r_{p}$ from $Z^{p}$ which is a contradiction with the definition of $a_{k}^{p}$. Consequently

$$
\kappa^{p} \leq \kappa^{p+1} \leq \kappa^{B\left(x_{0}, r_{0}\right)} .
$$

Now if $K$ is not separating in $B^{p}$, then there is $k_{1}$ and $k_{2}$, and there is a continuous path from $a_{k_{1}}^{p}$ to $a_{k_{2}}^{p}$ without meeting $K$. However we know that $K$ is separating in $B^{p+1}$ hence we get the contradiction.

Since all the $A_{k}^{p+1}$ are contained in a certain $\Omega_{l}$ and since $K$ is separating in $B^{p}$, we can deduce that every $A_{k}^{p}$ is also in an $\Omega_{l(k)}$. Moreover $l(k)$ is injective by (3.6) and by induction, so the conclusion follows.

\section{Whitney extension from a geometric function}

We still assume that $K$ is a closed set in $B\left(x_{0}, r_{0}\right)$ satisfying Hypothesis $H 1$ with a geometric function $\delta$, a minimal cone $Z$, a constant $\varepsilon_{0}<10^{-5}$ and a radius $\rho \in\left[\frac{1}{2} r_{0}, \frac{3}{4} r_{0}\right]$. Let $U>1$ be a constant that will be fixed later, depending on $C_{0}$ and a dimensional constant. In addition we assume that $\varepsilon_{0}$ is very small compared to $U^{-1}$. For all $t>0$ we define

$$
\mathcal{V}(t):=\bigcup_{x \in K \cap B(0, \rho)} B\left(x, \frac{t}{U} \delta(x)\right) .
$$


We also set

$$
\mathcal{V}:=\mathcal{V}(10)
$$

and

$$
z:=\mathcal{V}(30) \backslash \mathcal{V}\left(\frac{1}{10}\right)
$$

Finally we define

$$
\mathcal{V}_{\rho}:=\bigcup_{x ; B\left(x, \frac{10}{U} \delta(x)\right) \cap \partial B\left(x_{0}, \rho\right) \neq \emptyset} B\left(x, \frac{10}{U} \delta(x)\right) .
$$

Recall that by hypothesis, $K$ is separating in $B\left(x_{0}, r_{0}\right)$ and that for all $k \in\left[1, K^{B\left(x_{0}, r_{0}\right)}\right]$ we have denoted by $A_{k}\left(x_{0}, r_{0}\right)$ the connected components of $B\left(x_{0}, r_{0}\right) \backslash Z_{\varepsilon_{0} r_{0}}$ and by $\Omega_{k}\left(x_{0}, r_{0}\right)$ the connected component of $B\left(x_{0}, r_{0}\right) \backslash K$ that contains $A_{k}\left(x_{0}, r_{0}\right)$. We also set

$$
\Delta_{k}:=B\left(x_{0}, \rho\right) \cap\left(\Omega_{k}\left(x_{0}, r_{0}\right) \cup \mathcal{V}\right) .
$$

The purpose of this section is to prove the following lemma:

Lemma 4.1 (Whitney extension). There is a universal constant $c_{0}$ such that the following holds. Let $K$ be a closed set in $B\left(x_{0}, r_{0}\right)$ satisfying Hypothesis $H 1$ with a geometric function $\delta$, a minimal cone $Z$, a constant $\varepsilon_{0}<c_{0}$ and a radius $\rho \in\left[\frac{1}{2} r_{0}, \frac{3}{4} r_{0}\right]$. Then for all function $u \in W^{1,2}\left(B\left(0, r_{0}\right) \backslash K\right)$, and for all $k \in\left[1, \kappa^{B\left(x_{0}, r_{0}\right)}\right]$, there is a function

$$
v_{k} \in W^{1,2}\left(\Delta_{k} \backslash \mathcal{V}_{\rho}\right)
$$

such that

$$
v_{k}=u \text { in } B\left(x_{0}, \rho\right) \backslash \mathcal{V}
$$

and

$$
\int_{\Delta_{k} \backslash \mathcal{V}_{\rho}}\left|\nabla v_{k}\right|^{2} d x \leq \int_{\Delta_{k} \backslash \mathcal{V}\left(\frac{1}{3}\right)}|\nabla u|^{2} d x+C \int_{Z}|\nabla u|^{2} d x
$$

where $C$ is a constant depending only on dimension and where $\mathcal{V}, \mathcal{V}_{\rho}, Z$ and $\Delta_{k}$ are defined in (4.2), (4.4), (4.3) and (4.5) with a certain constant $U>30 C_{0}$.

Proof. We will use a Whitney type extension. To be clearer, two steps of the proof will be stated as Lemmas and will appear inside the proof of Lemma 4.1.

For all $x \in K \cap B\left(x_{0}, \rho\right)$ set

$$
B_{x}:=B\left(x, \frac{1}{U} \delta(x)\right)
$$

where $U \geq 30 C_{0}$ is a constant that will be fixed later. Then we choose a subfamily $\left\{W_{j}\right\}_{j \in J}$ of balls from $\left\{B_{x}\right\}$, maximal for the property that

$$
\forall j \neq j^{\prime}, \quad \frac{1}{100} W_{j} \cap \frac{1}{100} W_{j^{\prime}}=\emptyset .
$$


We denote by $r_{j}$ the radius of the ball $W_{j}$. The $\left\{W_{j}\right\}_{j \in J}$ is our Whitney family of balls, and we denote by $x_{j}$ and $r_{j}$ the center and radius of $W_{j}$. We have the following proprieties about the Whitney balls:

\section{Lemma 4.2.}

Whitney Property

Covering $\mathcal{V}$

The cover is bounded

Geometry is under control i) $10 W_{j} \cap 10 W_{j^{\prime}} \neq \emptyset \Rightarrow \frac{1}{20} r_{j^{\prime}} \leq r_{j} \leq 20 r_{j^{\prime}}$

ii) for all $t>0, \bigcup_{j \in J} t W_{j} \subset \mathcal{V}(t) \subset \bigcup_{j \in J}\left(20 t+\frac{3}{10}\right) W_{j}$

iii) $\exists C_{2} ; \forall x \in B\left(x_{0}, \rho\right), \sharp\left\{j \in J ; x \in 10 W_{j}\right\} \leq C_{2}$

Proof. We begin with i). Let $x \in B\left(x_{0}, \rho\right)$ and let $j$ and $k$ be two indices such that $10 W_{j}$ and $10 W_{k}$ contain $x$. Then if $x_{j}$ and $x_{k}$ are the centers of $W_{j}$ and $W_{k}$, since $\delta$ is $C_{0}$-lipschitz we have

$$
\begin{aligned}
\delta\left(x_{j}\right) & \leq \delta\left(x_{k}\right)+C_{0}\left|x_{k}-x_{j}\right| \\
& \leq U r_{k}+C_{0}\left|x_{k}-x\right|+C_{0}\left|x-x_{j}\right| \\
& \leq U r_{k}+10 C_{0} r_{k}+10 C_{0} r_{j} .
\end{aligned}
$$

Therefore,

$$
\left(U-10 C_{0}\right) r_{j} \leq\left(U+10 C_{0}\right) r_{k} .
$$

With the same argument exchanging $r_{j}$ and $r_{k}$ we can finally deduce that

$$
\frac{U-10 C_{0}}{U+10 C_{0}} r_{j} \leq r_{k} \leq \frac{U+10 C_{0}}{U-10 C_{0}} r_{j}
$$

thus i) follows if we consider that $U \geq 30 C_{0}$.

The first inclusion of ii) is trivial, by definition of $\mathcal{V}(t)$. So we have to prove the second inclusion. Let $y \in \mathcal{V}(t)$ and let $x$ be a point of $K$ such that $y \in t B_{x}:=$ $B\left(x, \frac{t}{U} \delta(x)\right)$. We denote by $r_{x}$ the radius of $B_{x}$. Since $\left\{W_{j}\right\}$ is a maximal family, there is a $j_{0}$ such that $\frac{1}{100} B_{x} \cap \frac{1}{100} W_{j_{0}} \neq \varnothing$ (otherwise we could add $B_{x}$ in the family $\left.\left\{W_{j}\right\}\right)$. Let $z$ be a common point. By the same argument as for i), we can deduce that $20^{-1} r_{j_{0}} \leq r_{x} \leq 20 r_{j_{0}}$. Now if $x_{j_{0}}$ is the center of $W_{j_{0}}$ we have

$$
\begin{aligned}
\left|y-x_{j_{0}}\right| & \leq|y-x|+|x-z|+\left|z-x_{j_{0}}\right| \\
& \leq t r_{x}+\frac{1}{100} r_{x}+\frac{1}{100} r_{j_{0}} \\
& \leq 20 t r_{j_{0}}+\frac{20}{100} r_{j_{0}}+\frac{1}{100} r_{j_{0}} \\
& \leq\left(20 t+\frac{3}{10}\right) r_{j_{0}}
\end{aligned}
$$

so $y \in\left(20 t+\frac{3}{10}\right) W_{j_{0}}$ and this proves the second inclusion of ii). 
For iii) it is just a simple consequence of a geometric fact in $\mathbb{R}^{N}$. Consider a family of balls in $\mathbb{R}^{N}$ that are all containing a same point, with radius equivalent to 1 and centered at distance more than $\frac{1}{100}$ to each other, then the number of these balls is finite. The proof of iii) follows.

Finally we have to prove iv). Let $j \in J$ and $r>0$ be such that $r_{j} \leq r \leq r_{0}$. By definition of $\delta$, we know that if $r \geq \delta\left(x_{j}\right)$, then $\beta\left(x_{j}, r\right) \leq \varepsilon_{0}$ and this is that we want. Now if $r_{j} \leq r \leq \delta\left(x_{j}\right)$ we have

$$
\beta\left(x_{j}, r\right) \leq \frac{\delta\left(x_{j}\right)}{r} \beta\left(x_{j}, \delta\left(x_{j}\right)\right) \leq U \varepsilon_{0}
$$

because $r \geq r_{j}=\frac{\delta\left(x_{j}\right)}{U}$, and iv) is proved.

We continue the proof of Lemma 4.1. With help of Lemma 3.1, for every $j \in J$ we can if necessary change $W_{j}$ to $2 W_{j}$ or $4 W_{j}$ in order to have that all the cones $Z^{j}$ associated to the $W_{j}$ in iv) are almost centered in $W_{j}$. To prove this, using a translation we can suppose that $W_{j}$ is centered at 0 . Then we apply Lemma 3.1 to the cone $Z$ associated to $W_{j}$, which is $U \varepsilon_{0} r_{j}$-close to $K$ in $W_{j}$. If $U \varepsilon_{0}<10^{-5}$, Lemma 3.1 says that we can choose among $W_{j}, 2 W_{j}$ or $4 W_{j}$, a ball such that $Z$ is almost centered. Since the cone $\tilde{Z}$ associated to this choice of ball is close to $Z$, it is also almost centered itself. This new family of balls still verify proprieties of Lemma 4.2 with constant that may be slightly different (by multiplying by 4).

Moreover, applying Lemma 3.4 to $U W_{j}$ we can assume that $W_{j}$ is well oriented.

We are now ready to make our Whitney extension $v$ from $u$ in $B\left(x_{0}, \rho\right)$. For every ball $W_{j}$ consider a function $\varphi_{j} \in C^{\infty}$, with compact support in $10 W_{j}$, equal to 1 on $8 W_{j}$ and to 0 out of $10 W_{j}$.

Lemma 4.3. There is a function $\varphi_{0} \in C^{\infty}$ such that

$$
\begin{aligned}
& \varphi_{0}=1 \quad \text { in } \quad B\left(x_{0}, \rho\right) \backslash \bigcup_{j \in J} 10 W_{j} \\
& \varphi_{0}=0 \quad \text { in } \quad \bigcup_{j \in J} 8 W_{j} \\
& \varphi_{0}+\sum_{j \in J} \varphi_{j} \geq 1 \quad \text { in } \quad B\left(x_{0}, \rho\right)
\end{aligned}
$$

and in addition there is a constant $C$ such that for all $j \in J$ and for all $x \in$ $10 W_{j} \backslash 8 W_{j}$,

$$
\left|\nabla \varphi_{0}(x)\right| \leq C \frac{1}{r_{j}} .
$$

Proof. Let $l \in C^{\infty}\left(\mathbb{R}^{+}\right)$such that $l$ is equal to 0 on $[0,8]$, equal to 1 on $[10,+\infty[$ and

$$
l^{\prime}(x) \leq 10 .
$$


Then the function

$$
\varphi_{0}(x):=\prod_{j \in J} l\left(\frac{\left|x-x_{j}\right|}{r_{i}}\right)
$$

satisfies the hypothesis of Lemma 4.3. Indeed, (4.7) and (4.8) is obvious from the definition of $\varphi_{0},(4.9)$ is easy to prove depending on a good construction of the $\varphi_{j}$, and (4.10) follows from (4.11), Property i) of Lemma 4.2 and also from the fact that the $\left\{10 W_{j}\right\}$ are in bounded cover with equivalent radius when they meet each other.

Let us now going back to the proof of Lemma 4.1. Set

$$
\theta_{j}=\frac{\varphi_{j}}{\varphi_{0}+\sum_{j \neq 0} \varphi_{j}}
$$

so that we have a partition of unity on $B\left(x_{0}, r_{0}\right)$. Since the $10 W_{j}$ are in bounded cover, the sum is locally finite.

In each $10 W_{j}$ there is a cone $Z^{j}$ such that

$$
K \cap 10 W_{j} \subset Z_{10 r_{j} U \varepsilon_{0}}^{j}
$$

with

$$
Z_{10 r_{j} U \varepsilon_{0}}^{j}:=\left\{y ; d\left(y, Z^{j}\right)<\varepsilon_{0} 10 U r_{j}\right\} .
$$

Denote by $A_{k}^{j}, 1 \leq k \leq k^{j}$ the connected components of $10 W_{j} \backslash Z_{r_{j} \varepsilon_{0}}^{j}$. Since the $10 W_{j}$ are well oriented, we know that each $A_{k}^{j}$ is contained in one of the big connected components $\Omega_{l(k)}$ of $B\left(x_{0}, r_{0}\right) \backslash K$. And we know that $l(k)$ is injective. Then we rename $A_{k}^{j}$ to $A_{l}^{j}$ such that $A_{l}^{j}$ is now contained in $\Omega_{l}^{j}$. By convention, if $l$ is such that $\Omega_{l}$ does not meet $W_{j}$, we set $A_{l}^{j}=\emptyset$. Hence for all $j$ and for $1 \leq k \leq k^{B\left(x_{0}, r_{0}\right)}$, we have defined $A_{k}^{j}$. In each nonempty $A_{k}^{j}$ we choose a point $a_{k}^{j} \in 10 W_{j} \backslash 8 W_{j}$ at greater distance than $7 r_{j}$ from $K$ and we also consider $D_{k}^{j}$ a ball centered at $a_{k}^{j}$ and of radius $\frac{1}{100} r_{j}$. We denote by $m_{k}^{j}$ the mean value of $u$ on $D_{k}^{j}$. It is always possible because the cones $Z^{j}$ are almost centered.

Now for all $k \in \mathbb{N} \cap\left[1, \kappa^{B\left(x_{0}, r_{0}\right)}\right]$ set

$$
v_{k}(x):=\varphi_{0}(x) u(x)+\sum_{j>0} m_{k}^{j} \theta_{j}(x) .
$$

The $v_{k}$ are well defined on $B\left(x_{0}, r_{0}\right) \backslash K$ and since $\bigcup_{j \in J} 10 W_{j} \subset \mathcal{V}$, the $v_{k}$ are equal to $u$ in the exterior of $\mathcal{V}$.

We want to check that the functions $v_{k}$ belong to $W^{1,2}\left(\Delta_{k} \backslash \mathcal{V}_{\rho}\right)$ and we want to estimate their energy with the energy of $u$. Let $x$ be a fixed point in $\bigcup_{j \in J} 10 W_{j}$ 
and call $J_{x}$ the set of all indices $j$ such that $10 W_{j}$ contains $x$. We know by Lemma 4.2 i) that all these balls have equivalent diameters.

On the other hand, all the $10 W_{j}$ for $j \in J_{x}$ are contained in $30 W_{j_{0}}$ where $j_{0}$ is any index fixed in $J_{x}$. By Property $v$ ) of Lemma 4.2, we know that there is a cone $Z_{x}$ containing $x_{j_{0}}$ (the center of $B_{j_{0}}$ ) and such that every point of $K \cap 30 B_{j_{0}}$ is at a distance less than $\varepsilon_{0} 120 U r_{j_{0}}$ from $Z_{x}$ where $r_{j_{0}}$ is the radius of $B_{j_{0}}$. We also know that $Z_{x}$ is almost centered in $B_{j_{0}}$. Thus $K$ is at a distance less than $400 U \varepsilon_{0} r_{j}$ from $Z_{x}$ in all the $W_{j}$ which contains $x$. Therefore, if we consider the connected components of $\mathbb{R}^{3} \backslash Z_{x}$, each one contains one and only one $D_{k}^{j}$ for all $j \in J_{x}$. Thus we can define for all $k$, a polyhedral domain $D_{k}^{x}$ that contains each $D_{k}^{j}$ for all $j \in J_{x}$. For instance we could define $D_{k}^{x}$ as being the convex hull of all the $D_{k}^{j}$ for $j \in J_{x}$. The important fact is that the Poincare Constant is uniformly bounded in all the $D_{k}^{x}$ (depending only on the radius of the $D_{k}^{j}$ ). Indeed, the diameter of $D_{k}^{x}$ is equivalent to $\frac{C}{U} \delta(x)$ because all the balls $W_{j}$ for $j$ in $J_{x}$ have a radius equivalent to $\frac{C}{U} \delta(x)$. Here the constant $C$ is just a geometric constant. Finally, $D_{k}^{x}$ does not meet $K$, and, as $\varepsilon_{0}$ is small enough, we also have

$$
D_{k}^{x} \subset \bigcup_{j \in J} 10 W_{j} \backslash 6 W_{j} \subset Z .
$$

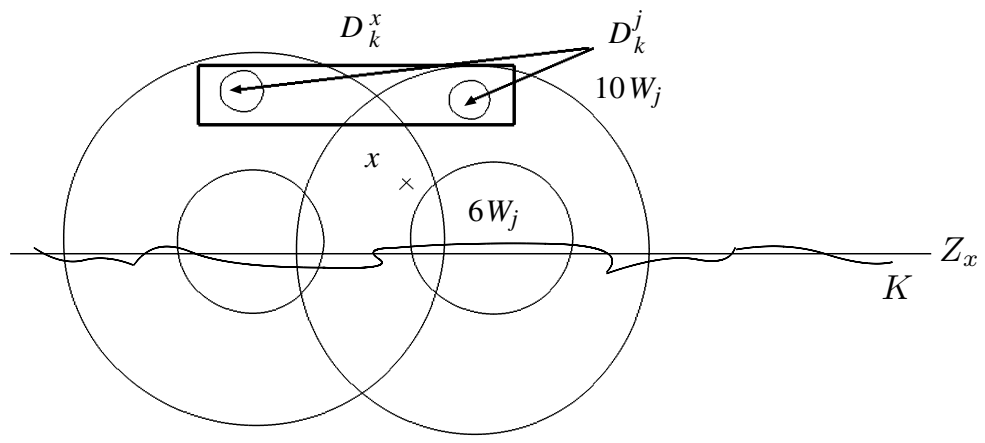

Figure 4.1. Picture of the situation when $Z_{x}$ is a hyperplane.

Let $m_{k}^{x}$ be the average of $u$ on $D_{k}^{x}$. Since the $\theta_{j}$ are a partition of unity, we know that $\sum \nabla \theta_{j}=0$ hence we can subtract $m_{k}^{x}$ and then

$$
\nabla v_{k}(x)=\theta_{0} \nabla u(x)+\nabla \theta_{0}\left(u-m_{k}^{x}\right)+\sum_{j>0}\left(m_{k}^{j}-m_{k}^{x}\right) \nabla \theta_{j}(x) .
$$

On the other hand using Poincaré inequality,

$$
\left|m_{k}^{j}-m_{k}^{x}\right| \leq C \frac{1}{r_{j}^{3}} \int_{D_{k}^{j}}\left|u(y)-m_{k}^{x}\right| d y \leq \frac{1}{r_{j}^{3}} \int_{D_{k}^{x}}\left|u(y)-m_{k}^{x}\right| d y \leq C \frac{1}{r_{j}^{2}} \int_{D_{k}^{x}}|\nabla u(y)| d y .
$$




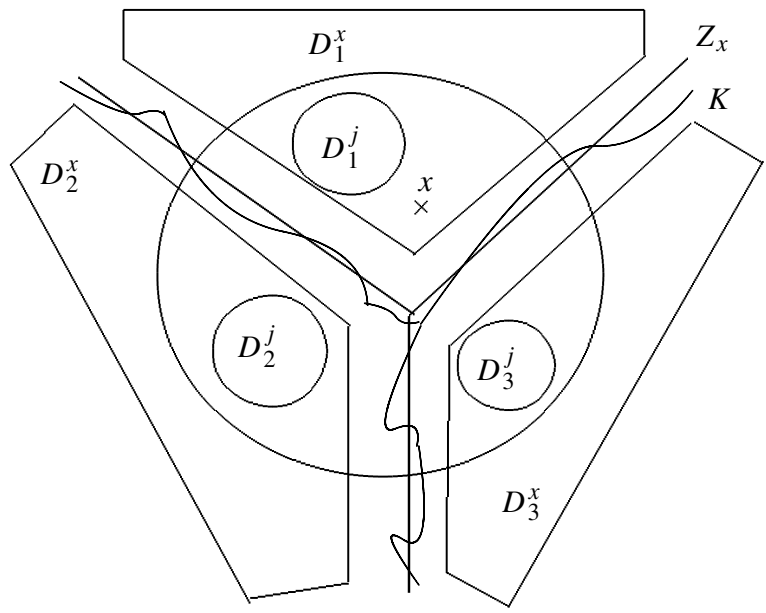

Figure 4.2. Picture of the situation when $Z_{x}$ is a $\mathbb{Y}$.

In addition all the $r_{j}$ for $j \in J_{x}$ are equivalent, and since $\left|\nabla \theta_{j}\right| \leq C r_{j}^{-1}$, every term in the sum in (4.12) is bounded by $C \frac{1}{r_{j}^{3}} \int_{D_{k}^{x}}|\nabla u(y)| d y$. Furthermore, $r_{j}$ is bounded from below by $C \delta(x)$ then finally since the sum has only $C_{2}$ terms,

$$
\left|\nabla v_{k}(x)\right| \leq \underbrace{|\nabla u(x)| \theta_{0}(x)}_{f_{1}}+\underbrace{C \frac{1}{\delta(x)^{3}} \int_{D_{k}^{x}}|\nabla u(y)| d y}_{f_{2}}+\underbrace{\left|\nabla \theta_{0}(x)\right|\left|u(x)-m_{k}^{x}\right|}_{f_{3}} .
$$

Next we write

$$
\left|\nabla v_{k}(x)\right|^{2} \leq C\left(f_{1}^{2}+f_{2}^{2}+f_{3}^{2}\right)
$$

and we will treat each $f_{i}$ successively. Let

$$
\Theta:=\left(\bigcup_{j \in J} 10 W_{j}\right) \backslash\left(\bigcup_{j \in J} 8 W_{j}\right) \subset z \quad \text { and } \quad \Theta_{k}:=\Theta \cap \Omega_{k}
$$

so that $\nabla \theta_{0}$ has its support in $\Theta$. It will be convenient to also define

$$
V:=\bigcup_{j \in J} 10 W_{j} \subset \mathcal{V}, \quad V_{k}:=V \cap \Omega_{k}
$$

and

$$
Q_{k}:=\bigcup_{x \in V} D_{k}^{x} \subset z
$$

We begin now by estimating $f_{1}$. Taking the integral on $x \in V$ immediately gives

$$
\int_{V} f_{1}^{2}(x) d x \leq \int_{\Theta_{k}}|\nabla u(x)|^{2} d x
$$


because $\theta_{0}=0$ on $V \backslash \Theta$. For $f_{2}$, applying Hölder's inequality we obtain

$$
f_{2}^{2} \leq C \frac{1}{\delta(x)^{3}} \int_{D_{k}^{x}}|\nabla u(y)|^{2} d y .
$$

Then integrating and applying Fubini leads to

$$
\begin{aligned}
\int_{V} f_{2}^{2}(x) d x & \leq C \int_{V}\left(\frac{1}{\delta(x)^{3}} \int_{D_{k}^{x}} \mathbf{1}_{Q_{k}}|\nabla u(y)|^{2} d y\right) d x \\
& \leq C \int_{Q_{k}}|\nabla u(y)|^{2}\left(\int_{\left\{x ; y \in D_{k}^{x}\right\}} \delta(x)^{-3} d x\right) d y .
\end{aligned}
$$

Now we claim that

$$
\int_{\left\{x ; y \in D_{k}^{x}\right\}} \delta(x)^{-3} d x \leq C .
$$

Here $C$ is a dimensional constant. As $x$ is fixed, $D_{k}^{x}$ is a polyhedral domain of diameter $\frac{C}{U} \delta(x)$ and at a distance less than $\frac{C}{U} \delta(x)$ from $x$. Hence $D_{k}^{x}$ is contained in a ball $B\left(x, \frac{C}{U} \delta(x)\right)$. Therefore, the set $\left\{x ; y \in D_{k}^{x}\right\}$ is contained in $A:=\left\{x ; d(x, y) \leq \frac{C}{U} \delta(x)\right\}$. We want to show that for all $x$ in $A, \delta(x)$ is equivalent to $\delta(y)$. This is where the choice of constant $U$ is important. Indeed, if $x$ is in $A$ then since $\delta$ is $C_{0}$-Lipschitz,

$$
\delta(x) \leq \delta(y)+C_{0}|x-y|
$$

hence

$$
\delta(y) \geq \delta(x)-\frac{C C_{0}}{U} \delta(x) .
$$

Recall that $U \geq 30 C_{0}$ is big as we want compared to $C_{0}$. Thus if $U$ is chosen large enough, $\frac{C C_{0}}{U}$ is less than 1 and then (4.15) gives

$$
\delta(x) \leq C \delta(y) .
$$

On the other hand,

$$
\delta(y) \leq \delta(x)+C_{0}|x-y| \leq C \delta(x) .
$$

Thus $\delta(x)$ and $\delta(y)$ are equivalent on $A$ and

$$
\int_{\left\{x ; y \in D_{k}^{x}\right\}} \delta(x)^{-3} d x \leq C \delta(y)^{-3} \int_{\{x ; d(x, y) \leq C \delta(y)\}} d x \leq C
$$

hence (4.14) is true and finally

$$
\int_{V} f_{2}^{2} d x \leq \int_{\Theta_{k}}|\nabla u(x)|^{2} d x+C \int_{Q_{k}}|\nabla u(y)|^{2} d y .
$$


We now come to the case of $f_{3}$, namely we want to estimate

$$
f_{3}:=\left|u(x)-m_{k}^{x}\right| \nabla \theta_{0}(x) .
$$

We may assume that $x$ is in the support of $\nabla \theta_{0}$, hence that $x \in \Theta_{k}$. We will use a Poincaré inequality and for this, it is fundamental that for all $x \in \Theta_{k}$ and for all $y \in D_{k}^{x}$, the segment $[x, y]$ does not meet the singular set $K$. Consequently, the next estimate is not true for all $x \in \Theta_{k}$, but it is true for all $x \in \Theta_{k} \backslash \mathcal{V}_{\rho}$. Indeed, if $10 W_{j_{0}}$ does not meet $\partial B\left(x_{0}, \rho\right)$, we claim that

$$
K \cap 10 W_{j_{0}} \subset K \cap \bigcup_{j \in J} \frac{6}{10} W_{j} .
$$

To see this, we denote $W_{j_{0}}:=B\left(x_{j_{0}}, r_{j_{0}}\right)$ and we use the fact that $\delta$ is $C_{0}$-lipschitz. Thus for all $x \in K \cap 10 W_{j_{0}}$ we have

$$
\delta(x) \geq \delta\left(x_{j_{0}}\right)-10 C_{0} r_{j_{0}} \geq U r_{j_{0}}-10 C_{0} r_{j_{0}} \geq 20 C_{0} r_{j_{0}}
$$

because remember that $U \geq 30 C_{0}$. Therefore, the last inequality together with Lemma 4.2 ii) yields

$$
\begin{aligned}
K \cap 10 W_{j_{0}} & \subset \bigcup_{x \in K \cap 10 W_{j_{0}}} B\left(x, \frac{3 C_{0}}{10 U} r_{j_{0}}\right) \\
& \subset \bigcup_{x \in K \cap 10 W_{j_{0}}} B\left(x, \frac{3}{200 U} \delta(x)\right) \subset \bigcup_{j \in J} \frac{6}{10} W_{j},
\end{aligned}
$$

and (4.16) follows. Note that (4.17) is not true if $W_{j_{0}} \cap \partial B\left(x_{0}, \rho\right) \neq \emptyset$ because $\delta(x)$ is not defined for $x$ outside $B\left(x_{0}, \rho\right)$. Denote by $J_{x}$ the set of indices such that $10 W_{j}$ contains $x \in \Theta_{k} \backslash \mathcal{V}_{\rho}$. For all $j \in J_{x}$ we know that $W_{j} \cap \partial B\left(x_{0}, \rho\right)=\varnothing$ by definition of $\mathcal{V}_{\rho}$. Now by applying (4.16) to all the $W_{j}$ with $j \in J_{x}$ it follows that

$$
K \cap \bigcup_{j \in J_{x}} 10 W_{j} \subset \bigcup_{j \in J} \frac{6}{10} W_{j}
$$

Therefore, using the definition of $\Theta_{k}$, passing to the complement and using the fact that all the $W_{j}$ for $j \in J_{x}$ are almost "aligned" on a same minimal cone $Z$, we deduce that for $\varepsilon_{0}$ small enough, the convex hull (denoted $\left.G_{x}\right)$ of $\Theta_{k} \cap\left(\bigcup_{j \in J_{x}} 10 W_{j}\right)$ does not meet $K$. Now since $D_{x}^{k} \subset G_{x}$ it follows that if $x \in \Theta_{k} \backslash \mathcal{V}_{\rho}$ and $y \in D_{k}^{x}$, the segment $[x, y]$ does not meet $K$, and moreover the Poincaré constant associated to the domain $G_{x}$ does not depend on $x$ in such a way that we have

$$
\int_{G_{x}}\left|u(y)-m_{k}^{x}\right| d y \leq C \delta(x) \int_{G_{x}}|\nabla u(y)| d y .
$$




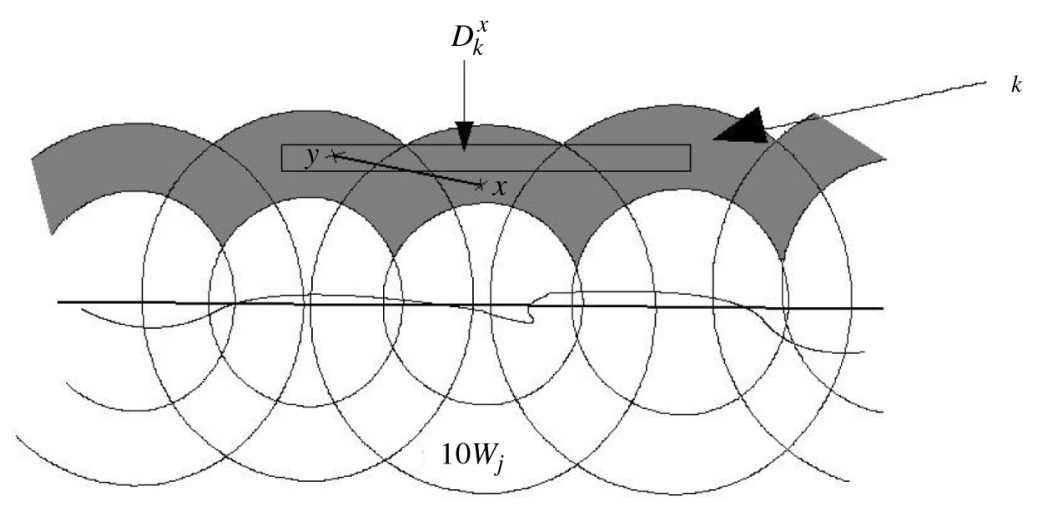

Figure 4.3.

Therefore, since for all $j$ and for any $x \in 2 W_{j}$ we have $m_{k}^{x}=m_{k}^{x_{j}}$ and $\delta(x) \simeq r_{j}$,

$$
\begin{aligned}
\int_{V_{k} \backslash \mathcal{V}_{\rho}}\left|u(x)-m_{k}^{x}\right|^{2}\left|\nabla \theta_{0}(x)\right|^{2} d x & \leq C \sum_{j \in J} \int_{10 W_{j}}\left|u(x)-m_{k}^{x}\right|^{2} \delta(x)^{-2} \mathbf{1}_{\Theta_{k} \backslash \mathcal{V}_{\rho}}(x) d x \\
& \leq C \sum_{j \in J} r_{j}^{-2} \int_{10 W_{j}}\left|u(x)-m_{k}^{x_{j}}\right|^{2} \mathbf{1}_{\Theta_{k} \backslash \mathcal{V}_{\rho}}(x) d x \\
& \leq C \sum_{j \in J ; W_{j} \cap \mathcal{V}_{\rho}=\emptyset} r_{j}^{-2} \int_{G_{x_{i}}}\left|u(x)-m_{k}^{x_{j}}\right|^{2} d x \\
& \leq C \sum_{j \in J ; W_{j} \cap \mathcal{V}_{\rho}=\emptyset} \int_{G_{x_{i}}}|\nabla u(x)|^{2} d x \\
& \leq C \int_{\Theta_{k}}|\nabla u(x)|^{2} d x .
\end{aligned}
$$

Then, since $\Theta_{k} \cup Q_{k} \subset Z$, putting (4.13), (4) and (4.19) together we have proved

$$
\int_{V \backslash \mathcal{V}_{\rho}}\left|\nabla v_{k}(x)\right|^{2} d x \leq C \int_{z}|\nabla u(x)|^{2} d x
$$

and this implies

$$
\begin{aligned}
\int_{\Delta_{k} \backslash \mathcal{V}_{\rho}}\left|\nabla v_{k}\right|^{2} & \leq \int_{\Delta_{k} \backslash\left(V \cup \mathcal{V}_{\rho}\right)}|\nabla u|^{2}+\int_{V \backslash \mathcal{V}_{\rho}}\left|\nabla v_{k}\right|^{2} \\
& \leq \int_{\Delta_{k} \backslash \mathcal{V}\left(\frac{1}{3}\right)}|\nabla u|^{2}+C \int_{z}|\nabla u|^{2}
\end{aligned}
$$

which ends the proof of Lemma 4.1. 


\section{Decay of energy in the complement of $\left(\varepsilon_{0}, \varepsilon\right)$-minimal sets}

We can now prove the main result.

Proof of Theorem 1.10. The argument is by "contradiction and compactness". The beginning is very close to [2, Theorem 8.19$]$. The main changes will come when we will have to prove Inequality (5.4).

Step 1 (Beginning of the proof). Let $\varepsilon_{0}:=c_{0}$ which is the constant given by Lemma 4.1 and suppose that Theorem 1.10 is false. Then there is an $i_{0} \in\{1,2,3\}$, a $\gamma<2(\sqrt{2}-1)$ and there is a positive radius $r<\frac{1}{2}$ such that for all $\varepsilon>0$ there is a triplet $\left(u_{\varepsilon}, E_{\varepsilon}, \rho_{\varepsilon}\right) \in \Lambda_{i_{0}}(\varepsilon)$ such that

$$
\int_{B(0, r) \backslash E_{\varepsilon}^{\rho}}\left|\nabla u_{\varepsilon}\right|^{2}>r^{2+\gamma} .
$$

In the following, $i_{0}$ is a fixed index. We call $Z^{0}$ a minimal cone of type $i_{0}$ centered at 0 . By rotation invariance we suppose that all the sets $E_{\varepsilon}$ are contained in $\{y \in$ $\left.B ; d\left(y, Z^{0}\right) \leq \varepsilon\right\}$.

For every positive number $a$ such that $0<a<10^{-5}$ we call $Z_{a}^{0}$ the region

$$
Z_{a}^{0}:=\left\{y \in B ; d\left(x, Z^{0}\right) \leq a\right\} .
$$

Then for $j \in\left[1, i_{0}+1\right] \cap \mathbb{N}$ we denote by $A_{j}$ the different connected components of $B \backslash Z_{a}^{0}$ and $m_{a, \varepsilon}^{j}$ the mean value of $u_{\varepsilon}$ on $A_{a}^{j}$. Since for all $\varepsilon$ we have

$$
\int_{B \backslash E_{\varepsilon}^{\rho}}\left|\nabla u_{\varepsilon}\right|^{2}=1,
$$

the Poincaré inequality says that for all $0<a<10^{-5}$ the sequences $u_{\varepsilon}-m_{10^{-5}, \varepsilon}^{j}$ are uniformly bounded for the norm $W^{1,2}\left(A_{a}^{j}\right)$. We also know that the sequence $\nabla u_{\varepsilon}$ is uniformly bounded in $L^{2}(B)$. Therefore, possibly extracting a further subsequence obtained by a diagonal argument, we may conclude that there is a subsequence $u_{n}$ that weakly converges in $W^{1,2}$ on all the $A_{a}^{j}$ to a function $u \in W^{1,2}\left(B \backslash Z^{0}\right)$, and such that $\nabla u_{n}$ also weakly converges in $L^{2}(B)$ to a certain function, which by uniqueness of limits in the distributional space is equal to $\nabla u$. We denote by $E_{n}$ the set associated to $u_{n}$, and also let $\varepsilon_{n}$ and $\rho_{n}$ be such that $\left(u_{n}, E_{n}, \rho_{n}\right) \in \Lambda\left(\varepsilon_{n}\right)$. In addition, passing if necessary to a subsequence, we can assume that $\rho_{n}$ converges to some real $\rho_{\infty} \in\left[\frac{1}{2}, \frac{3}{4}\right]$.

Step 2 ( $u$ is an energy minimizer). We claim that $u$ is an energy minimizing function in $B \backslash Z^{0}$, or in other words, $u$ is harmonic in $B \backslash Z^{0}$ and have zero normal derivative on both sides of $Z^{0}$. Let $\varphi$ be a $C^{\infty}$ function with compact support in $B \backslash Z^{0}$. Since for all $n$ we know that $u_{n}$ is an energy minimizer we have that

$$
\int_{B}\left\langle\nabla \varphi, \nabla u_{n}\right\rangle=0
$$


Then passing to the limit (because $\nabla u_{n}$ weakly converges in $L^{2}(B)$ ) we obtain

$$
\int_{B \backslash Z^{0}}\langle\nabla \varphi, \nabla u\rangle=0
$$

and this proves that $u$ is harmonic in $B \backslash Z^{0}$.

Now we want to show that $u$ has zero normal derivative on $Z^{0}$. So we have to show that for all $\varphi \in C_{0}^{1}(B)$,

$$
\int_{\Omega^{k}}\langle\nabla \varphi, \nabla u\rangle=0
$$

where $\Omega^{k}$ are connected components of $B \backslash Z^{0}$. For all $n$ we know that

$$
E_{n} \subset Z_{\varepsilon_{n}}^{0}:=\left\{y \in B ; d\left(y, Z^{0}\right) \leq \varepsilon_{n}\right\}
$$

and that $E_{n}$ is separating in $B$. Recall that $A_{\varepsilon_{n}}^{k}$ denotes the connected components of $B \backslash Z_{\varepsilon_{n}}^{0}$ and we call $\Omega_{n}^{k}$ the connected components of $B \backslash E_{n}$ that contains $A_{\varepsilon_{n}}^{k}$. Let $k_{0}$ be fixed and consider a sequence $c_{n}$ that converges to $+\infty$. Denote by $v_{n}$ the sequence in $W^{1,2}(B \backslash K)$ defined by

$$
v_{n}= \begin{cases}u_{n}+c_{n} & \text { in } \Omega_{n}^{k_{0}} \\ u_{n}-c_{n} & \text { in } \Omega_{n}^{k} \text { for } k \neq k_{0} \\ u_{n} & \text { in } B \backslash \bigcup_{k} \Omega_{n}^{k}\end{cases}
$$

Now let $\eta>0$ be fixed, and let $\chi(t)$ be a function on $\mathbb{R}$ such that for all $t, \chi^{\prime}(t) \leq \eta$ and such that $\lim _{t \rightarrow-\infty} \chi(t)=0$ and $\lim _{t \rightarrow+\infty} \chi(t)=1$. If $\varphi$ is a function in $C_{0}^{1}(B)$, the function $\varphi \chi\left(v_{n}\right) \in W^{1,2}\left(B \backslash E_{n}\right)$. Comparing energy of $u_{n}$ with energy of $u_{n}+\varepsilon_{n} \varphi \chi\left(v_{n}\right)$ we obtain that

$$
\liminf _{n \rightarrow \infty}\left[\int_{B} \varphi\left\langle\nabla u_{n}, \nabla \chi\left(v_{n}\right)\right\rangle+\int_{B} \chi\left(v_{n}\right)\left\langle\nabla u_{n}, \nabla \varphi\right\rangle\right] \geq 0 .
$$

On the other hand, $\nabla \chi\left(v_{n}\right)=\chi^{\prime}\left(v_{n}\right) \nabla u_{n},\left|\chi^{\prime}\right| \leq \eta$ and $\int_{B}\left|\nabla u_{n}\right|^{2}=1$. Moreover, $\chi\left(v_{n}\right)$ strongly converges in $L^{2}(B)$ to $\mathbf{1}_{\Omega^{k} 0}$. We deduce

$$
\int_{\Omega^{k_{0}}}\langle\nabla u, \nabla \varphi\rangle \geq-\eta\|\varphi\|_{\infty}
$$

and we conclude by the fact that $\eta$ is an arbitrary constant. We do the same for all $\Omega^{k}$ and this proves that $u$ is an energy minimiser in $B \backslash Z^{0}$. Hence, we know by Section 2 that for all $r \in] 0,1]$,

$$
\frac{1}{r^{2}} \int_{B(0, r) \backslash Z^{0}}|\nabla u|^{2} \leq r^{2(\sqrt{2}-1)} \int_{B(0,1) \backslash Z^{0}}|\nabla u|^{2}=r^{2(\sqrt{2}-1)} .
$$


Therefore, if we show that $\nabla u_{n}$ strongly converges in $L^{2}(B(0, r))$, the contradiction will follow by passing to the limit in (5.1). The purpose of all the rest of the proof is to justify this convergence.

Step 3 (The limit measure $\mu$ and its regular part). We consider the measures $\mu_{n}:=$ $\left|\nabla u_{n}\right|^{2} d x$ on $B$. Since for all $n$ we have $\mu_{n}(B)=1$, passing if necessary to a subsequence we may assume that $\mu_{n}$ weakly converge to some measure $\mu$. Since $\nabla u_{n}$ weakly converge in $L^{2}$ to $\nabla u$, all we can say is that (see for instance [2, Proposition 1.62(b)])

$$
|\nabla u|^{2} d x \leq \mu
$$

So we have to show that $(5.3)$ is an equality in $B(0, r)$. This would be enough because the Radon-Riesz Theorem says that if a sequence $f_{n}$ of $L^{p}$ functions weakly converge to some function $f$, and if in addition the sequence of $L^{p}$ norms $\left\|f_{n}\right\|_{p}$ converge to $\|f\|_{p}$ then $f_{n}$ strongly converge to $f$ in $L^{p}$.

First of all, let us show that the regular part of $\mu$ with respect to Lebesgue measure is $|\nabla u|^{2} d x$ and that the singular part is concentred on $Z^{0}$. To show that, let us recall that $u_{n}$ is a sequence of harmonic functions with $L^{2}$ norms uniformly bounded and then are uniformly bounded for the $L^{\infty}$ norm on all compact sets of $B \backslash Z^{0}$. Thus by covering $B \backslash Z^{0}$ with a countably union of compact sets and by using a diagonal argument we can say that $u_{n}$ converges to $u$ for the $L^{p}$ norm on all the compact sets and for all $p$. Moreover since the $u_{n}$ are harmonic we know that their gradients are also uniformly bounded. Then extracting a further subsequence we may assume that $\nabla u_{n}$ converges to $\nabla u$ in $L^{2}$ strongly on all compact sets of $B \backslash Z^{0}$. It follows that for every compact set $U$ in $B \backslash Z^{0}$,

$$
\mu(U)=\lim _{n \rightarrow+\infty} \mu_{n}(U)=\lim _{n \rightarrow+\infty} \int_{U}\left|\nabla u_{n}\right|^{2} d x=\int_{U}|\nabla u|^{2} d x
$$

Step 4 (Strategy for the next steps). Consequently, to prove that $\mu=|\nabla u|^{2} d x$ everywhere, we have to show that $\mu\left(Z^{0}\right)=0$. So we consider a domain $\mathcal{C}_{h}$ containing $K$. We define the base

$$
G:=B\left(0, \rho_{\infty}\right) \cap Z^{0}
$$

and then set

$$
\mathcal{C}_{h}:=\{y \in B ; d(y, G) \leq h\} \cap B\left(0, \rho_{\infty}\right) .
$$

Define also

$$
\mathcal{C}_{h}^{n}:=\left\{y \in B ; d\left(y, G_{n}\right) \leq h\right\} \cap B\left(0, \rho_{n}\right)
$$

based on

$$
G_{n}:=B\left(0, \rho_{n}\right) \cap Z^{0}
$$

such that $\mathcal{C}_{h}^{n}$ converges to $\mathcal{C}_{h}$ for the Hausdorff distance. The strategy is to estimate $\mu\left(\mathcal{C}_{h}\right)$ and then let $h$ tend to 0 . 
Let us sketch the next ideas that will be used to finish the proof. The main point is to prove an inequality of the following type

$$
\int_{\mathcal{C}_{h}}\left|\nabla u_{n}\right|^{2} d x \leq C \int_{S_{n}}\left|\nabla u_{n}\right|^{2} d x
$$

where $S_{n}$ is a closed set such that $E_{n}^{\rho_{n}} \cap B\left(0, \rho_{n}\right) \subset B\left(0, \rho_{n}\right) \backslash S_{n}$ and $S_{n}$ converges to

$$
S_{\infty}:=\left\{x \in \mathcal{C}_{h} ; d\left(x, Z^{0}\right) \geq C_{5} d\left(x, \partial \mathcal{C}_{h}\right)\right\} .
$$

If (5.4) is true, then passing to the limit (that will be justify later),

$$
\mu\left(\mathcal{C}_{h}\right) \leq C \mu\left(S_{\infty}\right)=\int_{S_{\infty}}|\nabla u|^{2} d x \leq C h\|\nabla u\|_{\infty}^{2}
$$

and then letting $h$ tend to 0 we conclude that $\mu\left(Z^{0} \cap B\left(0, \rho_{\infty}\right)\right)=0$.

Now the purpose of the following is to justify the above arguments. To prove (5.4) we will use the Whitney extension of preceding section.

Step 5 (Construction of geometric function $\delta$ ). Let $n$ be fixed. We have to construct a function $\delta$ in order to apply Lemma 4.1 to $u_{n}$ and $E_{n}$. In particular, $\delta$ will depend on $n$ but we don't make it explicit to lighten the notations.

Let $\left\{B_{i}\right\}$ be the family of balls in the definition of $\left(\varepsilon_{0}, \varepsilon_{n}\right)$-minimality of $E_{n}$. For all $i \in I$ denote by $\psi_{i}$ a positive function, 1-lipschitz, such that

$$
\psi_{i}(x):=\left\{\begin{array}{ll}
r_{i} & \text { in } B_{i} \\
0 & \text { out of } 2 B_{i}
\end{array} .\right.
$$

Then for all $x \in B$, let $d(x)$ be defined by

$$
d(x):=\sum_{i \in I} \psi_{i}(x)
$$

The sum is locally finite so $d(x)$ is $C_{0}$-Lipschitz where $C_{0}$ is the constant from the bounded cover propriety of $\left\{2 B_{i}\right\}_{i \in I}$.

Then for all $x \in B(0,1)$ we define

$$
\delta(x):=\max \left(d\left(x, \partial \mathcal{C}_{h}^{n}\right), d(x)\right) .
$$

By construction and by definition of $\left(\varepsilon_{0}, \varepsilon_{n}\right)$-minimality of $E_{n}$, we can deduce that $E_{n}$ is satisfying Hypothesis $H 1$ in $B(0,1)$ with geometric function $\delta$, constant $\varepsilon_{0}$ and radius $\rho_{n}$. Therefore applying Lemma 4.1 , we obtain for all $k \in \mathbb{N} \cap\left[1, k^{B(0,1)}\right]$, a function $v_{k} \in W^{1,2}\left(B\left(0, \rho_{n}\right) \cap\left(\Omega_{k} \cup \mathcal{V}^{n}\right) \backslash \mathcal{V}_{\rho_{n}}^{n}\right)$ with

$$
\mathcal{V}^{n}:=\bigcup_{x \in K \cap B\left(0, \rho_{n}\right)} B\left(x, \frac{10}{U} \delta(x)\right)
$$


and

$$
\mathcal{V}_{\rho_{n}}^{n}:=\bigcup_{x ; B\left(x, \frac{10}{U} \delta(x)\right) \cap \partial B\left(0, \rho_{n}\right) \neq \emptyset} B\left(x, \frac{10}{U} \delta(x)\right)
$$

and where $U$ is a constant satisfying $U \geq 30 C_{0}$.

Step 6 (Construction of a competitor and key estimate). By construction, the $v^{k}$ are equal to $u_{n}$ in $B(0,1) \backslash \mathcal{V}^{n}$. Moreover, if $\varepsilon$ is small enough, $\left(\mathcal{V} \backslash \mathcal{V}_{\rho_{n}}\right) \cap B\left(0, \rho_{n}\right) \subset$ $\mathcal{C}_{h}^{n}$ and note that we can fix $C_{1}$ in such a way that

$$
\mathcal{V}_{\rho_{n}}^{n} \subset \bigcup_{i \in I_{\rho_{n}}} C_{1} B_{i}
$$

(see (1.9) for the definition of $C_{1}$ ). Indeed to prove (5.5) let $x$ be a point in $\mathcal{V}_{\rho_{n}}^{n}$ and $y$ be such that $B\left(y, \frac{10}{U} \delta(y)\right) \cap \partial B\left(0, \rho_{n}\right) \neq \emptyset$ and such that $x \in B\left(y, \frac{10}{U} \delta(y)\right)$. Set $r_{y}:=\frac{10}{U} \delta(y)$. Since $\delta(y)=\max \left(d(y), d\left(y, \partial \mathcal{C}_{h}^{n}\right)\right)$ and since $U \geq 30 C_{0}$, we can deduce that $\delta(y)=d(y):=\sum_{i \in I} \psi_{i}(y)$. Therefore there is an index $i_{0}$ such that $\psi_{i_{0}}(y)$ is not equal to zero. This implies that $y \in 2 B_{i_{0}}$. We can also suppose that $i_{0}$ is the index for which $\psi_{i_{0}}(y)$ is the maximum of all the $\psi_{i}(y)$. On the other hand, $r_{y}=\frac{1}{U} \sum_{i ; y \in 2 B_{i}} \psi_{i}(y) \leq \frac{C_{0}}{U} \psi_{i_{0}}(y) \leq \frac{C_{0}}{U} r_{i_{0}}$ (because the $\left\{2 B_{i}\right\}$ are in bounded cover with constant $C_{0}$ ). So we have proved

$$
B\left(y, r_{y}\right) \subset\left(2+10 \frac{C_{0}}{U}\right) B_{i_{0}}
$$

and (5.5) follows if we choose $C_{1}=\left(2+10 \frac{C_{0}}{U}\right)$.

Lemma 4.1 gives

$$
\int_{\mathcal{V}^{n} \backslash \mathcal{V}_{\rho_{n}}^{n}}\left|\nabla v^{k}\right|^{2} d x \leq C \int_{Z^{n}}|\nabla u|^{2} d x
$$

with $z^{n}$ defined by (4.3). Therefore we can compare the energy of $u_{n}$ with the energy of $v$ defined by

$$
v=v^{k} \text { in } B\left(0, \rho_{n}\right) \cap \Omega_{k} .
$$

On other components we can assume that $v=0$. Recall that $u_{n}$ is by definition the energy minimizing function in $B \backslash E_{n}^{\rho_{n}}$ and note that $v$ is a competitor in $B\left(0, \rho_{n}\right)$ that is equal to $u_{n}$ out of $\mathcal{V}^{n}$ thus

$$
\int_{\mathcal{V}^{n} \backslash E_{n}^{\rho_{n}}}\left|\nabla u_{n}\right|^{2} \leq \sum_{k=1}^{k} \int_{\mathcal{V}^{n} \backslash \mathcal{V}_{\rho_{n}}^{n}}\left|\nabla v^{k}\right|^{2} \leq C \int_{Z^{n}}\left|\nabla u_{n}\right|^{2} .
$$

Hence

$$
\begin{aligned}
\int_{\mathcal{C}_{h}}\left|\nabla u_{n}\right|^{2} & \leq \int_{\mathcal{C}_{h} \backslash \mathcal{V}^{n}}\left|\nabla u_{n}\right|^{2}+\int_{\mathcal{V}^{n}}\left|\nabla u_{n}\right|^{2} \\
& \leq \int_{\mathcal{C}_{h} \backslash \mathcal{V}^{n}}\left|\nabla u_{n}\right|^{2}+C \int_{Z^{n}}\left|\nabla u_{n}\right|^{2} .
\end{aligned}
$$


Now we define the closed set

$$
S_{n}:=\mathcal{C}_{h} \backslash \stackrel{\circ}{\mathcal{V}}^{n} \cup z^{n}
$$

Then we have proved

$$
\int_{\mathcal{C}_{h}}\left|\nabla u_{n}\right|^{2} \leq C \int_{S_{n}}\left|\nabla u_{n}\right|^{2} .
$$

Step 7 (Convergence of $S_{n}$ ). The point is now to show that

$$
S_{n} \text { converges to } S_{\infty}:=\left\{x \in \mathcal{C}_{h} ; d\left(x, Z^{0}\right) \geq C_{5} d\left(x, \partial \mathcal{C}_{h}\right)\right\}
$$

and

$$
\limsup _{n \rightarrow+\infty}\left(\int_{S_{n}}\left|\nabla u_{n}\right|^{2} d x\right) \leq \mu\left(S_{\infty}\right) .
$$

Here $C_{5}$ is a constant that will be chosen later.

We begin with (5.8). For all $\eta>0$ we denote by $S_{\infty}^{\eta}$ a $\eta$-neighborhood of $S_{\infty}$. In other words

$$
S_{\infty}^{\eta}:=\left\{x \in B ; d\left(x, S_{\infty}\right)<\eta\right\} .
$$

We want to show that for $n$ big enough and if the constant $C_{5}$ is chosen properly, then all the $S_{n}$ are contained in $S_{\infty}^{\eta}$. So let $n_{0}$ be fixed in such a way that for all $n \geq n_{0}$ we have $\varepsilon_{n}<\eta$ and $\left|\rho-\rho_{n}\right| \leq \eta$. Hence, for all $n \geq n_{0}$ and for all $x \in E_{n}$ we have $d\left(x, Z^{0}\right) \leq \eta$. Let now $x$ be a point in $S_{n}$ for $n \geq n_{0}$. We claim that $x$ is in $S_{\infty}^{C_{7} \eta}$ for a certain constant $C_{7}$. Indeed, if $x$ is in $S_{n}$, then by definition of $z^{n}$ and $v^{n}$ (see (4.2) and (4.3)) $x$ does not belong to any of the $B\left(y, \frac{1}{10 U} \delta(y)\right)$. In other words,

$$
x \in \mathcal{C}_{h} \backslash \mathcal{V}(1 / 10)_{n}
$$

with

$$
\mathcal{V}(t)_{n}:=\bigcup_{y \in E_{n} \cap B\left(0, \rho_{n}\right)} B\left(y, \frac{t}{U} \delta(y)\right) .
$$

Denote by

$$
\mathcal{V}(1 / 10)_{n}^{\eta}:=\left\{y \in \mathcal{C}_{h} ; d\left(y, \mathcal{V}(1 / 10)_{n}\right) \leq C_{6} \eta\right\}
$$

with $C_{6}$ a constant to be fixed later. We want to show that there is a constant $c$ (depending on $C_{0}$ and $U$ ) such that

$$
\mathcal{V}(1 / 10)_{n}^{\eta} \supseteq\left\{y \in \mathcal{C}_{h} ; d\left(y, Z^{0}\right) \leq c d\left(y, \partial \mathcal{C}_{h}\right)\right\} .
$$

Let $y \in \mathcal{C}_{h}$ be such that $d\left(y, Z^{0}\right) \leq c d\left(y, \partial \mathcal{C}_{h}\right)$. We call $\bar{y} \in Z^{0}$ a point such that

$$
d(y, \bar{y})=d\left(y, Z^{0}\right) .
$$

Since $E_{n}$ is separating and since $E_{n} \subset Z_{\varepsilon_{n}}^{0}$, there is a point $z \in E_{n}$ such that

$$
d(\bar{y}, z) \leq \eta
$$


otherwise the ball $B\left(\bar{y}, \varepsilon_{n}\right)$ would not contain any point of $E_{n}$. Since $\delta$ is $C_{0^{-}}$ lipschitz,

$$
\begin{aligned}
\delta(y) & \leq \delta(z)+C_{0}|y-z| \\
& \leq \delta(z)+C_{0}(|y-\bar{y}|+|\bar{y}-z|) \\
& \leq \delta(z)+C_{0}\left(c d\left(y, \partial \mathcal{C}_{h}\right)+\eta\right) .
\end{aligned}
$$

Recall that

$$
\delta(y)=\max \left(d\left(y, \partial \mathcal{C}_{h}^{n}\right), d(y)\right) \geq d\left(y, \partial \mathcal{C}_{h}^{n}\right) \geq d\left(y, \partial \mathcal{C}_{h}\right)-\eta .
$$

Hence,

$$
\left(1-c C_{0}\right) d\left(y, \partial \mathcal{C}_{h}\right)-\left(1+C_{0}\right) \eta \leq \delta(z)
$$

Now,

$$
\begin{aligned}
|y-z| & \leq|y-\bar{y}|+|\bar{y}-z| \\
& \leq c d\left(y, \partial \mathcal{C}_{h}\right)+\eta \\
& \leq c\left(1-c C_{0}\right)^{-1}\left[\delta(z)+\left(1+C_{0}\right) \eta\right]+\eta .
\end{aligned}
$$

Then we can choose $c<1$ small enough in order to have

$$
|y-z| \leq \frac{1}{10 U} \delta(z)+C_{6} \eta
$$

where $C_{6}$ is depending on $c$. This implies that $y \in B\left(z, \frac{1}{10 U} \delta(z)+C_{6} \eta\right)$, thus (5.10) is proved. Then by passing (5.10) to the complementary we deduce

$$
\mathcal{C}_{h} \backslash \mathcal{V}(1 / 10)_{n}^{\eta} \subset S_{\infty}
$$

from which we easily get

$$
\mathcal{C}_{h} \backslash \mathcal{V}(1 / 10)_{n} \subset S_{\infty}^{C_{\supset} \eta}
$$

and this proves (5.8).

Step 8 (Strong convergence and end of the proof). It is time now to prove (5.9) and to finish the proof of the theorem. We keep the same notations as before. That is for all $\eta$, we know that there is an integer $n_{0}$ such that for all $n \geq n_{0}, S_{n} \subset S_{\infty}^{C_{6} \eta}$. By (5.7) we know that

$$
\int_{\mathcal{C}_{h}}\left|\nabla u_{n}\right|^{2} d x \leq C \int_{S_{n}}\left|\nabla u_{n}\right|^{2} d x .
$$

In other words if $\mu_{n}$ is the measure $\left|\nabla u_{n}\right|^{2} d x$ we have

$$
\mu_{n}\left(\mathcal{C}_{h}\right) \leq C \mu_{n}\left(S_{n}\right) .
$$


On the other hand, since $\mu_{n}$ weakly converges to $\mu$ and since $\mathcal{C}_{h}$ is a fixed closed set, we can deduce that $\mu_{n}\left(\mathcal{C}_{h}\right)$ converges to $\mu\left(\mathcal{C}_{h}\right)$. Now since $S_{n}$ is included in $S_{\infty}^{\eta}$ for $n$ big enough,

$$
\mu\left(\mathcal{C}_{h}\right) \leq C \liminf _{n \rightarrow \infty} \mu_{n}\left(S_{\infty}^{\eta}\right)
$$

thus since now $S_{\infty}^{\eta}$ does not depend on $n$, we can apply the weak convergence and get

$$
\mu\left(\mathcal{C}_{h}\right) \leq C \mu\left(S_{\infty}^{\eta}\right)
$$

Finally, letting $\eta$ tend to 0 we obtain

$$
\mu\left(\mathcal{C}_{h}\right) \leq C \mu\left(S_{\infty}\right)
$$

This last limit is justified because $S_{\infty}$ is the decreasing intersection of $S_{\infty}^{\eta}$.

To finish the proof of the theorem we use the fact that $\mu$ is regular with respect to the Lebesgue measure on $S_{\infty}$ so (5.11) gives

$$
\mu\left(\mathcal{C}_{h}\right) \leq C \int_{S_{\infty}}|\nabla u|^{2} d x \leq C h\|\nabla u\|_{\infty}^{2}
$$

We know that $\nabla u$ is bounded on $S_{\infty}$ because $u$ is an harmonic function with Neumann condition on $Z^{0}$.

Now by letting $h$ tend to 0 we obtain that $\mu\left(Z^{0} \cap B\left(0, \rho_{\infty}\right)\right)=0$ and this gives that $\nabla u_{n}$ strongly converges to $\nabla u$ in $L^{2}\left(B\left(0, \rho_{\infty}\right)\right)$. So passing to the limit in (5.1) we get a contradiction with (5.2) and this proves the theorem.

\section{References}

[1] L. Ambrosio, N. FusCo and D. Pallara, Partial regularity of free discontinuity sets II, Ann. Scuola Norm. Sup. Pisa Cl. Sci. (4) 24 (1997), 39-62.

[2] L. Ambrosio, N. Fusco and D. Pallara, "Fonctions of Bounded Variation and Free Discontinuity Problems", Oxford University Press, 2000.

[3] A. Bonnet, On the regularity of edges in image segmentation, Ann. Inst. H. Poincaré, Anal. Non Linéaire 13 (1996), 485-528.

[4] M. Dauge, "Elliptic Boundary Value Problems on Corner Domains", Vol. 1341, Lecture Notes in Mathematics, Springer-Verlag, Berlin, 1988. Smoothness and asymptotics of solutions.

[5] M. Dauge, Neumann and mixed problems on curvilinear polyhedra, Integral Equations Operator Theory 15 (1992), 227-261.

[6] G. DAVID, $C^{1+\alpha}$-regularity for two dimensional almost-minimal sets in $\mathbb{R}^{n}$, J. Geom. Anal., to appear.

[7] G. DAVID, "Singular Sets of Minimizers for the Mumford-Shah Functional", Birkhäuser Verlag, 2005.

[8] G. David, T. De Pauw and T. Toro, A generalisation of Reifenberg's theorem in $\mathbb{R}^{3}$, Geom. Funct. Anal. 18 (2008), 1168-1235.

[9] A. LEMENANT, Regularity of the singular set for Mumford-Shah minimizers in $\mathbb{R}^{3}$ near a minimal cone, preprint, 2008. 
[10] A. Lemenant, "Sur la régularité des minimiseurs de Mumford-Shah en dimension 3 et supérieure", Thesis Université Paris Sud XI, Orsay, 2008.

[11] E. R. REIFENBERG, Solution of the plateau problem for $m$-dimensional surfaces of varying topological type, Acta Math. 104 (1960), 1-92.

[12] J. E. TAYLOR, The structure of singularities in soap-bubble-like and soap-film-like minimal surfaces, Ann. of Math. 103 (1976), 489-539.

Université Paris XI

Bureau 15 Bâtiment 430

91400 Orsay, France

antoine.lemenant@math.u-psud.fr 